\title{
Tracking the Genetic Susceptibility Background of B-Cell Non-Hodgkin's Lymphomas from Genome-Wide Association Studies
}

\author{
Isaias Hernández-Verdin ${ }^{1,2}$, Karim Labreche ${ }^{1,2}$, Marion Benazra 1,2,3,4,5 , Karima Mokhtari ${ }^{6,7}$, \\ Khê Hoang-Xuan 1,2,3,4,7,8 and Agusti Alentorn 1,2,3,4,7,*
}

1 Faculté de Médecine, Sorbonne Université, 75013 Paris, France; isaias.hernandez@icm-institute.org (I.H.-V.); karim.labreche@icm-institute.org (K.L.); marion.benazra@icm-institute.org (M.B.);

khe.hoang-xuan@aphp.fr (K.H.-X.)

2 Brain and Spine Institute (ICM), 75013 Paris, France

3 National Institute of Health and Medical Research (Inserm) U 1127, 75013 Paris, France

4 National Center for Scientific Research, Joint Research Unit 7225, 75013 Paris, France

5 Brain and Spine Institute (ICM), iGenSeq Platform, 75013 Paris, France

6 Raymond Escourolle Department of Neuropathology, Public Assistance-Hospitals of Paris, Hospital Group of Pitié-Salpêtrière, 75013 Paris, France; karima.mokhtari@aphp.fr

7 Assistance Publique Hôpitaux de Paris (APHP), Department of Neurology-2, Groupe Hospitalier Pitié Salpêtrière, 75013 Paris, France

8 Réseau Expert National LOC (Lymphomes Oculo-Cérébraux), Groupe Hospitalier Pitié Salpêtrière, 75013 Paris, France

* Correspondence: agusti.alentorn@aphp.fr

Citation: Hernández-Verdin, I.;

Labreche, K.; Benazra, M.;

Mokhtari, K.; Hoang-Xuan, K.;

Alentorn, A. Tracking the Genetic Susceptibility Background of B-Cell Non-Hodgkin's Lymphomas from Genome-Wide Association Studies. Int. J. Mol. Sci. 2021, 22, 122. https:// dx.doi.org/10.3390/ijms22010122

Received: 5 November 2020 Accepted: 18 December 2020 Published: 24 December 2020

Publisher's Note: MDPI stays neutral with regard to jurisdictional claims in published maps and institutional affiliations.

Copyright: () 2020 by the authors. Licensee MDPI, Basel, Switzerland. This article is an open access article distributed under the terms and conditions of the Creative Commons Attribution (CC BY) license (https: / / creativecommons.org/ licenses/by/4.0/).

\begin{abstract}
B-cell non-Hodgkin's lymphoma (NHL) risk associations had been mainly attributed to family history of the disease, inflammation, and immune components including human leukocyte antigen (HLA) genetic variations. Nevertheless, a broad range of genome-wide association studies (GWAS) have shed light into the identification of several genetic variants presumptively associated with B-cell NHL etiologies, survival or shared genetic risk with other diseases. The present review aims to overview HLA structure and diversity and summarize the evidence of genetic variations, by GWAS, on five NHL subtypes (diffuse large B-cell lymphoma DLBCL, follicular lymphoma FL, chronic lymphocytic leukemia CLL, marginal zone lymphoma MZL, and primary central nervous system lymphoma PCNSL). Evidence indicates that the HLA zygosity status in B-cell NHL might promote immune escape and that genome-wide significance variants can give biological insight but also potential therapeutic markers such as WEE1 in DLBCL. However, additional studies are needed, especially for non-DLBCL, to replicate the associations found to date.
\end{abstract}

Keywords: B-cell non-Hodgkin's lymphoma; GWAS; cancer risk; HLA

\section{Introduction}

Malignant lymphomas are among the most common head and neck neoplasm from lymphoreticular system origin and can be defined as Hodgkin's or non-Hodgkin's lymphoma (NHL), in which approximately $25 \%$ arises from extra-nodal locations like Waldeyer's ring, oral cavity, salivary glands, thyroid, larynx, nasal cavity, paranasal sinuses, skin, brain, eyes, leptomeninges, or spinal cord [1,2]. According to the 2016 World Health Organization classification there are around 60 distinct subtypes of NHL with diffuse large B-cell lymphoma (DLBCL, about 30\%), follicular lymphoma (FL, about 20\%) and chronic lymphocytic leukemia/small lymphocytic leukemia (CLL/SLL) among the most common [3-5]. Along with the wide location distribution of the lymphomas, this group of diseases has varying etiologies and prognosis, for example the five-year survival is $85 \%$ for CLL, $80 \%$ for $\mathrm{FL}, 76.5 \%$ for marginal zone lymphoma (MZL), but $<50 \%$ for more aggressive lymphomas such as DLBCL or even $30 \%$ for primary central nervous system lymphoma (PCNSL) [6-8]. Furthermore, 
there have been vast population studies to associate the presence of different etiologies, such as smoking, height, weight, autoimmune conditions, alcohol consumption, viral infections, and genetics, with the risk of developing any subtype of NHL $[3,9,10]$. Nevertheless, despite these efforts, there are only few established risk factors including autoimmune conditions (e.g., Sjögren disease, rheumatoid arthritis, systemic lupus erythematosus, and multiple sclerosis), immunodeficiency syndromes, organ transplants, breast implants and specific infections (e.g., Helicobacter pylori for mucosa-associated lymphoid tissue lymphoma of the stomach, immunodeficiency virus, and mononucleosis) [11-13].

More recently, sequencing technologies like next generation sequencing (NGS) and genome-wide association studies (GWAS), have broaden the possible candidates by using thousands of genetic variants for multiple genetic risk factors identification [14]. GWAS combining the population structure (Q) jointly with the genetic marker based kinship matrix (K) mixed linear model, also called linear mixed model, where the test statistic for significance is drawn from the central Chi-square distribution by comparing the allele frequencies of the cases to the controls. A variant is said to be significant at genome-wide level if the $p$ value is $\leq 5 \times 10^{-8}$, which was set by taking a 0.05 significance level and roughly dividing by the total number of independent blocks of linked genes in Europeans (thought to be 1,000,000) [7,15]. Regarding GWAS within the B-cell NHL context, most studies have focused on genetic variants at chromosome $6 \mathrm{p} 21$, specifically human leukocyte antigen (HLA) variants, since that region is critical for innate and adaptive immune responses, but there have been also efforts to find associations with variants outside this chromosome and other etiologies [7,10,11,16-19].

In this review, we give an overview of HLA structure and diversity, and then we summarize the most recent GWAS presented in five B-cell NHL subtypes: DLBCL, FL, CLL, MZL, and PCNSL in the light of loci (HLA and others) diversity and zygosity specific associations in addition to further clinical evaluations when suitable. Furthermore, we present the genetic overlap between B-cell NHL subtypes and autoimmune diseases, height, lipid traits, or other lymphomas.

\section{HLA Overview}

The association found between Hodgkin's lymphoma (HL) and HLA-B gene variation allowed to the later discovery that the major histocompatibility complex (MHC) is the genomic region with the highest number of associated human diseases [20,21]. The MHC, a hyper gene-dense region located at chromosome 6p21.3, encodes a set of 21 proteincoding loci that gives rise to three different types of HLA molecules. Firstly, class I, encoded by the highly polymorphic $H L A-A, H L A-B$, and HLA-C genes ("classical"); but also $H L A-E, H L A-F$, and HLA-G genes ("nonclassical") both of which are composed of a single $\alpha$ chain non-covalently bound to a small $\beta 2$-microglobulin polypeptide encoded by another chromosome (15q21). Secondly, class II, encoded by the HLA-DPA1, HLA-DQA1, HLA-DQA2, HLA-DRA, HLA-DPB1, HLA-DQB1, HLA-DQB2, HLA-DRB1, HLA-DRB2, HLA$D R B 3, H L A-D R B 4$, and $H L A-D R B 5$ genes which are composed of $\alpha-\beta$ heterodimer. Thirdly, class III, encoded by 61 genes (ex. MIC, SKI2W) involved in inflammation, leukocyte maturation and complement cascade [7,22-24]. These HLA genes comprise approximately four million base pair region, giving rise to more than 15,000 different classical HLA class I and II alleles which can, theoretically, serve for presenting over $10^{12}$ different peptides if the antigen-presenting cell (APC) is heterozygous at each of the six classical class I or II HLA loci $[25,26]$.

Nucleated cells express HLA class I molecules where small peptides (8-10 amino acids) are bound to the $\alpha 1-\alpha 2$ domains at the HLA peptide-binding site for later recognition by $\alpha \beta$ T-cell receptors (TCRs) on CD8+ T cells. On the other hand, monocytes/macrophages, dendritic cells and B cells express HLA class II receptors which can present larger peptides (13-25 amino acids) to TCRs on CD4+ T cells, hence inducing the orchestrated immune response against the pathogen due to cytokines releasing either by helping $B$ cells to secrete high affinity antibodies or by inducing macrophage activation [7,22,23]. Furthermore, 
HLA class I molecules can bind to natural killer (NK) cells through immunoglobulin-like receptors and C-type lectin-like CD94/NKG2 receptors (Figure 1) [27].

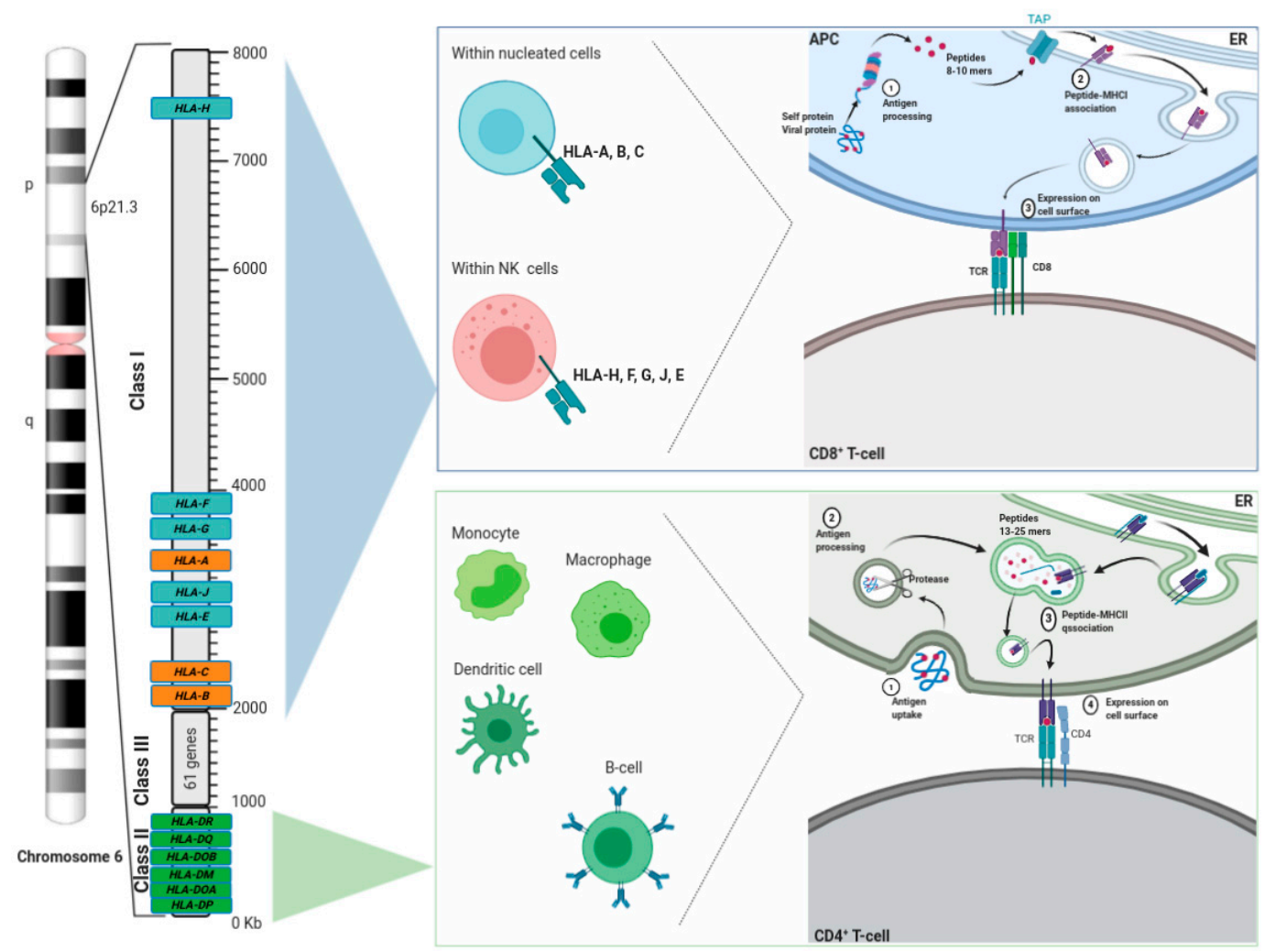

Figure 1. Schematic map of the human leukocyte antigen (HLA) genomic region showing the distribution of HLA genes along with the summarized mechanism of antigen presentation.

Along with the extreme gene density and polymorphism at the MHC locus, linkage disequilibrium, low-throughput methodologies and samples sizes made HLA-disease associations complicated. However, with the advent of both high-throughput wholegenome-based methodologies (example GWAS) and the evolution of big data analysis, researchers can measure the contribution of a single genetic variation across the genome on a disease risk by leveraging linkage disequilibrium [15].

\section{GWAS in B-Cell NHL}

\section{1. $D L B C L$}

Representing around $30 \%$ of NHL and affecting preferentially older adults, diffuse Large B-cell lymphoma is the most common type of NHL. During the last two decades, treatment with immunochemotherapy consisting of cyclophosphamide, doxorubicin, vincristine, and prednisolone combined with the anti-CD20 monoclonal antibody rituximab (R-CHOP) has become the gold standard. This regimen results in a cure rate of $60 \%$ and a five-year survival rate of $60 \%$ for germinal center B-cell (GCB) subtype or $35 \%$ for activated $\mathrm{B}$-cell $(\mathrm{ABC})$ subtype; however, clinical course is heterogeneous, even after elucidation of cell of origin (ABC or $\mathrm{GCB}$ ), thus requiring new biomarkers for elucidating patient outcome and for adapting treatment strategies [28,29]. Different risks factors have been identified. A stepwise logistic regression meta-analysis of 4667 cases and 22,639 controls, found that DLBCL is associated with B-cell activating autoimmune diseases (odds ratio $[\mathrm{OR}]=2.36,95 \%$ confidence interval $(\mathrm{CI})=1.80$ to 3.09$)$, hepatitis $C$ virus seropositivity $(\mathrm{OR}=2.02,95 \% \mathrm{CI}=1.47$ to 2.76$)$, family history of NHL (OR $=1.95,95 \% \mathrm{CI}=1.54$ to 2.47 ), and higher young adult body mass index $(\mathrm{OR}=1.58,95 \% \mathrm{CI}=1.12$ to 2.23 , for $35+\mathrm{vs} 18.5$ to $22.4 \mathrm{~kg} / \mathrm{m}^{2}$ ). Conversely, different potential presumptive protective factors have been 
proposed, such as higher sun exposure $(\mathrm{OR}=0.78$ and $0.80,95 \% \mathrm{CI}=0.69$ to 0.89 and 0.71 to $0.90)$, in two studies, and lifetime alcohol consumption (OR $=0.57,95 \% \mathrm{CI}=0.44$ to 0.75 , for $>400 \mathrm{~g}$ vs nondrinker) in one study. Vitamin D deficiency has been suggested as negative prognostic factor in patients with aggressive DLBCLs but was not found to be associated with dietary intake $(\mathrm{OR}=1.03,95 \% \mathrm{CI}=0.90$ to 1.19$)$, hence indicating that other factors rather than vitamin D may be involved [30,31]. More recent studies evaluating DLBCL risk, one using lipid trait variants in 2661 cases and 6221 controls found positive association with high density lipoproteins (OR $=1.14 ; 95 \%$ CI, 1.00-1.30), while another study evaluating height as variable; however, neither of them were significant after adjusting for multiple testing $[10,18]$.

HLA-B (rs2523607) locus has been associated with DLBCL risk, initially described in a GWAS study with 3857 cases $/ 7766$ controls from European population $(\mathrm{OR}=1.32$; $\left.95 \% \mathrm{CI}=1.21-1.44 ; p=2.40 \times 10^{-10}\right)$ and then reported in 1124 patients and 3596 controls from Asian population $\left(\mathrm{OR}=3.05 ; 95 \% \mathrm{CI}=1.32-7.05 ; p=9.0 \times 10^{-3}\right)$, though not reaching genome-wide significance [32,33] (Table 1). Regarding pleiotropy of DLBCL with other diseases, a European population study found that variant rs10484561 (HLADQB1*01:01 DQA1*01:01 DQB1*05:01 extended haplotype, Linkage Disequilibrium (LD) $\left.\mathrm{r}^{2}=1.0\right)$ is associated with both DLBCL risk $\left(\mathrm{OR}=1.36 ; 95 \% \mathrm{CI}=1.21-1.52 ; p=1.40 \times 10^{-7}\right)$ and $\mathrm{FL}$ risk $\left(\mathrm{OR}=1.64 ; 95 \% \mathrm{CI}=1.45-1.86 ; p=5.0 \times 10^{-15}\right)$ using independent cohorts [34]. Additionally, a GWAS of 3857 DLBCL cases and 7666 controls used previously systemic lupus erythematosus (SLE) associated loci to evaluate the risk of DLBLC, finding HLA risk allele rs1270942. Another study evaluated multiple sclerosis (MS) and rheumatoid arthritis (RA) with DLBCL risk, but not genome-wide significance was reached [11,19]. Moreover, HLA homozygosity was found to be associated with increased DLBCL risk for HLA-B, HLA-C and HLA-DRB1 alleles among Europeans [35].

GWAS have been also widely used for non-HLA alleles associations, more remarkably susceptibility risk have been found in different studies for: PVT1 (rs4733601 and rs13255292) in three different GWAS studies in which one also found to be associated with MS risk $\left(p=5 \times 10^{-8}\right)$; EXOC2 (rs116446171) [11,31,32] and CD86 (rs2681416 and rs9831894) [32,36]. PVT1 is a non-coding RNA affecting MYC activation, a driver gene in lymphomas; EXOC2 functions at the interface between host defense and cell death regulation and CD86 is well known for its role in T-cell activation [32,37]. Other, implicated loci are 2p23.3 (NCOA1), 3p24.1 (EOMES-AZI2), 5q31.3 (ARAP3) and 3q27 (BCL6-LPP); interestingly the BCL6 has been vastly documented to be involved in B-cell lymphomagenesis due to its role as critical regulator of germinal centers and rs6773363 (EOMES-AZI2) is indirectly involved in the activation of the NF- $\kappa$ B signaling pathway $[32,36,38]$. Another study $(399$ DLBCL cases and 4243 controls) of Japanese population, identified risk for a variant within intron 3 of $C D C 42 B P B\left(\mathrm{OR}=3.5 ; 95 \% \mathrm{CI}=2.13-5.88 ; p=3.30 \times 10^{-7}\right)$, a gene with cell migration and cytoskeletal reorganization functions, a variant on $L N X 2(\mathrm{OR}=1.43$; 95\% CI = 1.23-1.67; $\left.p=6.57 \times 10^{-6}\right)$, which indirectly mediates the NOTCH signaling and variant on POU6F2 $\left(\mathrm{OR}=1.57 ; 95 \% \mathrm{CI}=1.32-1.88 ; p=7.05 \times 10^{-7}\right)$, a transcriptional regulator [39]. In addition to PVT1, other overlapping risk variants for DLBCL and MS were rs1270942 (RDBP), rs3130557 (PSORS1C1), and rs2425752 (NCOA5) [11].

Another GWAS approach, using 491 DLBCL WGS data (31\% discovery cohort; 69\% validation cohort) and 1000 control WGS data, found NF-kB pathway activation by $3^{\prime}$ cis-regulatory mutations on NFKBIZ but only on ABC DLBCL subtype which was later correlated with increasing expression on different DLBCL cell lines when compared to the non-mutated ones. GCB subtype, on the other hand, was associated with poor overall survival for FCGR2B over expressing patients $\left(\mathrm{HR}=2.18 ; p=5.7 \times 10^{-3}\right)$ [40]. Furthermore, though it has not been fully explored, some studies have shown that the presence of activation-induced cytidine deaminase (AICDA) targeting motifs (WRC/GYW) within different point mutations, for example provoking induced translocations of PD-L1/PD-L2 with PIM1, TP63 and IGH or changes on the general mutational signatures across germinal center subtypes [40-42]. 
GWAS studies with survival data provided some evidence to finding potential prognostic or therapeutic targets in DLBCL, for example, a two-stage French study comprising four different cohorts in European population led to the discovery of two non-coding variants. The first one was rs7712513 at 5q23.2 (near SNX2 and SNCAIP). The second one was rs7765004 at 6q21 (near MARCKS and HDAC2) that reached genome-wide significance for overall-survival association but not for progression free survival (PFS; Table 2). SNX2 expression is reduced in human colorectal carcinoma and has been identified as a fusion partner of ABL1 in B-cell acute lymphoblastic leukemia; meanwhile, SNCAIP has been only reported in medulloblastoma studies. On the other hand, MARCKS has been widely studied for its role in invasion, proliferation, and drug resistance within different types of cancers [28,43-45]. Another study using data recovered from the Genome Expression Omnibus (GEO) from 1804 DLBCL patients and performed a guilt-by-association analysis of only the 500 top-ranked CD20-associated gene probes. This study found WEE1, a replication checkpoint kinase that arrests cells at the G2/M checkpoint to give time for DNA repair, and PARP1, a repairing protein involved in high genomic instability and NF-kB activation, as potential candidates for DLBCL treatments. They further evaluated these targets using inhibiting drugs (AZD1775 for WEE1 and olaparib for PARP1) on different cell lines finding increased cytotoxic effects. Furthermore, a later study from the same group led to the discovery that combined WEE1 and anti-apoptotic protein inhibition enhances premature mitotic entry and DNA damage which may benefit genomic unstable DLBCL cells $[17,46]$. Moreover, there are over 20 clinical trials exploring adavosertib, the most potent and selective WEE1 inhibitor, as a single agent or in combination for different indications (clinicaltrials.gov, October 2020).

\section{2. $F L$}

Follicular lymphoma is an indolent B-cell malignancy with higher five-year survival than DLBCL, though a subset of tumors can transform into more aggressive forms of lymphomas. FL is characterized by variable clinical outcomes, multiple relapses, and risk associations that includes family history of NHL (OR $=1.99 ; 95 \% \mathrm{CI}=1.55$ to 2.54$)$ and greater body mass index (OR $=1.15 ; 95 \% \mathrm{CI}=1.04$ to 1.27 per $5 \mathrm{~kg} / \mathrm{m}(2)$ increase) [47-49]. Two different three-stage GWAS studies in European populations, found that variant rs10484561 is associated with $\mathrm{FL}$ risk $\left(\mathrm{OR}_{1}=1.95 ; \mathrm{OR}_{2}=1.64 ; 95 \% \mathrm{CI}_{1}=1.72-2.22 ; 95 \% \mathrm{CI}_{2}=1.45-1.86\right.$; $p<1 \times 10^{-8}$ ) which, in addition, was later found to be implicated in DLBCL risk and in complete linkage disequilibrium with the HLADRB1*01:01 ${ }^{\sim}$ DQA1*01:01 DQB1*05:01 haplotype $\left(\right.$ LD- $\left.\mathrm{r}^{2}=1.0\right)$. Additional HLA-DQB1 variants associated with FL risk were, rs7755224 and rs2647012. The first one was in complete LD with variant rs10484561 suggesting that the effect was related to this. The last one was found 962 base pairs away from rs10484561. However, they were not in $\mathrm{LD}\left(\mathrm{r}^{2}<0.1\right)$ and the association was protective and genome-wide significant after mutual adjustment (rs2647012-OR $=0.70 ; 95 \%$ $\left.\mathrm{CI}=0.67-0.78 ; p=4 \times 10^{-12} ; \mathrm{rs} 10484561-\mathrm{OR}=1.64 ; 95 \% \mathrm{CI}=1.45-1.86 ; p=5 \times 10^{-15}\right)$, suggesting a totally different evolutionary origin $[34,50]$. This last variant was later found on additional studies in different populations (Caucasians and Chinese) at genome-wide significance [51,52]. Additional protective variants, found in another study with 699 cases and 2222 controls, were rs9275517 $\left(\mathrm{OR}=0.63 ; 95 \% \mathrm{CI}=0.55-0.73 ; p=4.03 \times 10^{-11}\right)$ and rs3117222 ( $\left.\mathrm{OR}=0.66 ; 95 \% \mathrm{CI}=0.57-0.77 ; p=1.45 \times 10^{-7}\right)$; furthermore, the second variant was correlated with higher HLA-DPB1 expression in lymphoblastoid cell lines by using mRNA expression from MuTHER and Gen Cord datasets [53]. In 2014, Skibola et al. identified two variants within the HLA-II class to be significantly associated with increased FL risk $\left(\mathrm{rs} 12195582-\mathrm{OR}=1.78 ; 95 \% \mathrm{CI}=1.68-1.88 ; p=5.36 \times 10^{-100} ; \mathrm{rs} 17203612-\mathrm{OR}=1.43\right.$; $95 \%$ CI $=1.32-1.57 ; p=4.59 \times 10^{-16}$ ) [54]. In addition to pleiotropy with DLBCL, HLA variants associated at genome-wide significance with FL has also been found for SLE, specifically two variants at HLA-DOB allele (rs1894406 and rs2071475) and one at HLADRB1 allele (rs9271775) [11]. In respect to homozygosity, HLA-DRB1 and HLA-DRQ1 alleles were found to be associated with increased FL risk [35]. 


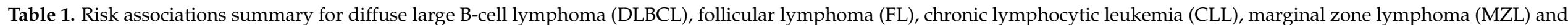
primary central nervous system lymphoma (PCNSL) with different loci identified by genome-wide association studies (GWAS).

\begin{tabular}{|c|c|c|c|c|c|c|c|c|c|}
\hline Study & Year & Race/Ethnicity & \#Cases/\#Controls & SNP/Alteration & Chr & Gene(s) & OR $(95 \% \mathrm{CI})$ & $p$-Value & Reference \\
\hline \multirow{19}{*}{ DLBCL risk } & 2009 & European & $783 / 3377$ & rs6457327 & $6 p 21.33$ & PSORS1 * & $1.69(1.43-2.00)$ & $7.0 \times 10^{-5}$ & [54] \\
\hline & 2011 & European & $1592 / 6581$ & rs10484561 & $6 p 21.32$ & $H L A-D Q B 1$ & $1.36(1.21-1.52)$ & $1.4 \times 10^{-7}$ & [34] \\
\hline & \multirow{4}{*}{2011} & \multirow{4}{*}{ Asian } & \multirow{4}{*}{$399 / 4243$} & rs751837 & $14 q 32$ & $C D C 42 B P B$ & $3.5(2.127-5.88)$ & $3.3 \times 10^{-7}$ & \multirow{4}{*}{ [39] } \\
\hline & & & & rs7097 & $13 q 12$ & $L N X 2$ & $1.437(1.23-1.67$ & $6.5 \times 10^{-6}$ & \\
\hline & & & & rs4551233 & 7 & POU6F2 & $1.57(1.32-1.88)$ & $7.05 \times 10^{-7}$ & \\
\hline & & & & rs4443228 & 4 & - & $2.43(1.70-3.45)$ & $7.03 \times 10^{-7}$ & \\
\hline & \multirow{6}{*}{2014} & \multirow{6}{*}{ European } & \multirow{6}{*}{$3857 / 7666$} & rs2523607 & $6 p 21.33$ & $H L A-B$ & $1.32(1.21-1.44)$ & $2.40 \times 10^{-10}$ & \multirow{6}{*}{ [32] } \\
\hline & & & & rs116446171 & $6 \mathrm{p} 25.3$ & EXOC2 * & $2.20(1.87-2.59)$ & $2.33 \times 10^{-21}$ & \\
\hline & & & & rs79480871 & $2 p 23.3$ & NCOA1 & $1.34(1.21-1.49)$ & $4.23 \times 10^{-8}$ & \\
\hline & & & & rs13255292 & \multirow{2}{*}{$8 \mathrm{q} 24.21$} & \multirow{2}{*}{ PVT1 } & $1.22(1.15-1.29)$ & $9.98 \times 10^{-13}$ & \\
\hline & & & & rs4733601 & & & $1.18(1.11-1.25)$ & $3.63 \times 10^{-11}$ & \\
\hline & & & & rs79464052 & $5 q 31.3$ & ARAP3 & $1.34(1.21-1.49)$ & $5.57 \times 10^{-8}$ & \\
\hline & \multirow{3}{*}{2015} & \multirow{3}{*}{ Asian } & \multirow{3}{*}{$1124 / 3596$} & rs116446171 & $6 \mathrm{p} 25.3$ & EXOC2 * & $2.04(1.63-2.56)$ & $3.9 \times 10^{-10}$ & \multirow{3}{*}{ [33] } \\
\hline & & & & rs13255292 & $8 \mathrm{q} 24.21$ & PVT1 & $1.34(1.19-1.52)$ & $2.1 \times 10^{-6}$ & \\
\hline & & & & rs2523607 & $6 \mathrm{p} 21.33$ & $H L A-B$ & $3.05(1.32-7.05$ & $9 \times 10^{-3}$ & \\
\hline & 2018 & \multirow{2}{*}{ European } & \multirow{2}{*}{$3617 / 8753$} & Homozygosity & $6 p 21.33$ & $H L A-B, H L A-C$ & $1.31(1.06-1.60)$ & $8 \times 10^{-4}$ & \multirow{2}{*}{ [35] } \\
\hline & 2018 & & & Homozygosity & & $H L A-D R B 1$ & $2.10(1.24-3.55)$ & $1 \times 10^{-4}$ & \\
\hline & \multirow[b]{2}{*}{2019} & \multirow[b]{2}{*}{ European } & $5662 / 9237$ & rs9831894 & $3 q 13.33$ & $C D 86^{*}, I L D R 1 *$ & 0.83 & $3.62 \times 10^{-13}$ & \multirow[b]{2}{*}{ [36] } \\
\hline & & & $5510 / 12,817$ & rs6773363 & $3 p 24.1$ & $\underset{*}{E O M E S}{ }_{*}^{*}, A Z I 2$ & 1.20 & $2.31 \times 10^{-12}$ & \\
\hline
\end{tabular}


Table 1. Cont.

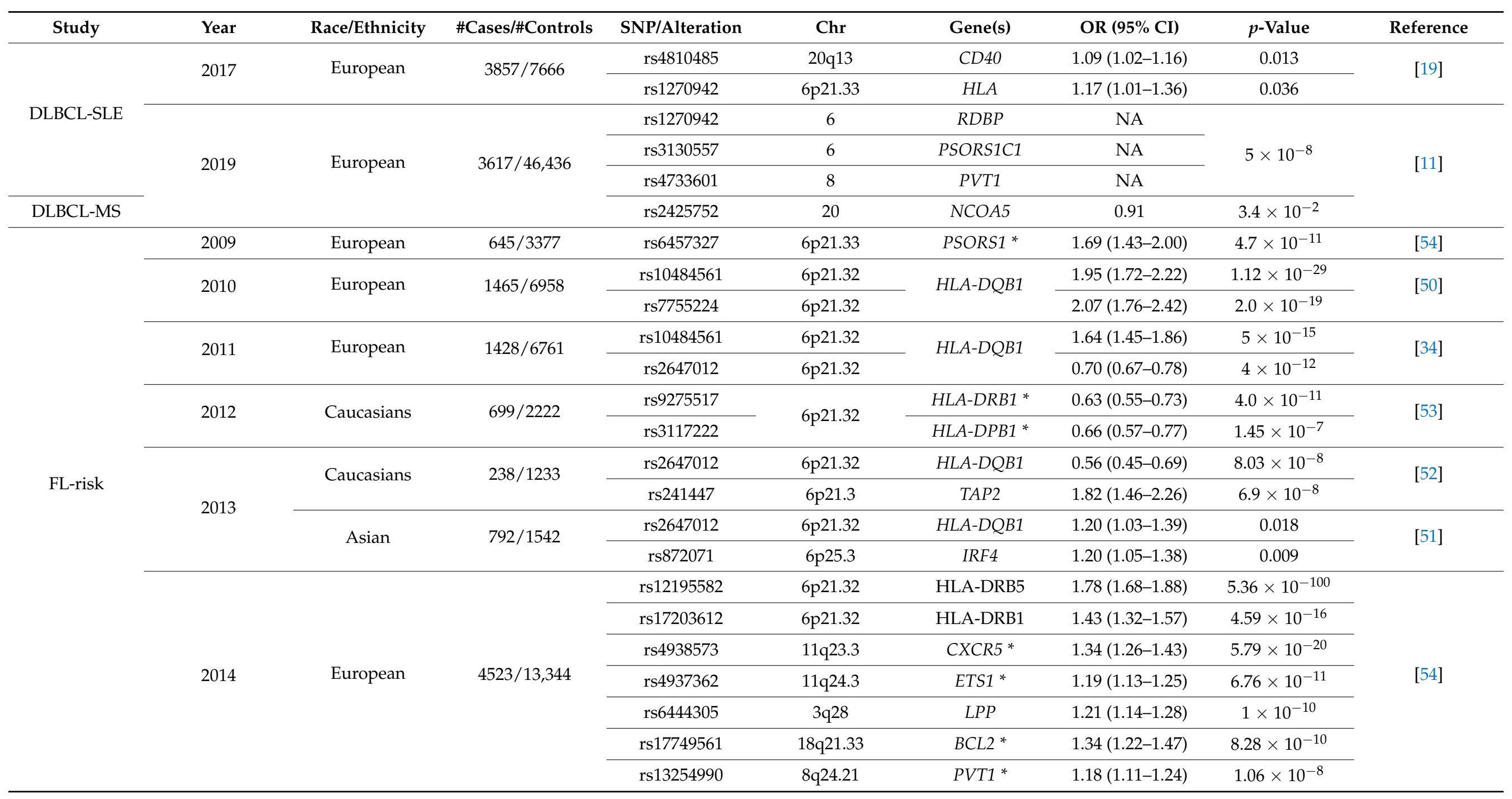


Table 1. Cont.

\begin{tabular}{|c|c|c|c|c|c|c|c|c|c|}
\hline Study & Year & Race/Ethnicity & \#Cases/\#Controls & SNP/Alteration & Chr & Gene(s) & OR $(95 \%$ CI $)$ & $p$-Value & Reference \\
\hline \multirow{4}{*}{ FL-risk } & \multirow[b]{2}{*}{2014} & \multirow[b]{2}{*}{ European } & \multirow[b]{2}{*}{$4523 / 13,344$} & rs3751913 & $17 q 25.3$ & CYBC1 & $1.23(1.14-1.33)$ & $2.24 \times 10^{-7}$ & \multirow[b]{2}{*}{ [54] } \\
\hline & & & & rs2681416 & $3 q 13.33$ & CD86 & $1.16(1.09-1.22)$ & $2.33 \times 10^{-7}$ & \\
\hline & \multirow{2}{*}{2018} & \multirow{2}{*}{ European } & \multirow{2}{*}{$2686 / 8753$} & Homozygosity & $6 \mathrm{p} 21.32$ & HLA-DRB1 & $1.54(1.31-1.82)$ & $1 \times 10^{-4}$ & \multirow{2}{*}{ [35] } \\
\hline & & & & Homozygosity & $6 \mathrm{p} 21.32$ & $H L A-D Q B 1$ & $1.42(1.23-1.65)$ & $1 \times 10^{-4}$ & \\
\hline FL-DLBCL & 2011 & European & $1428 / 6581$ & rs2647012 & $6 \mathrm{p} 21.32$ & $H L A-D Q B 1$ & 1.36 & $1.4 \times 10^{-7}$ & [50] \\
\hline \multirow{9}{*}{ FL-SLE } & \multirow{9}{*}{2019} & \multirow{9}{*}{ European } & \multirow{9}{*}{$2686 / 46,436$} & rs1015166 & 6 & TAP2 & NA & \multirow{9}{*}{$5 \times 10^{-8}$} & \multirow{9}{*}[11]{} \\
\hline & & & & rs1894406 & 6 & $H L A-D O B$ & NA & & \\
\hline & & & & rs2071475 & 6 & $H L A-D O B$ & NA & & \\
\hline & & & & rs2072634 & 6 & $C F B$ & NA & & \\
\hline & & & & rs2293861 & 6 & MSH5 & NA & & \\
\hline & & & & rs7774197 & 6 & TNXB & NA & & \\
\hline & & & & rs9271775 & 6 & $H L A-D R B 1$ & NA & & \\
\hline & & & & rs4938573 & 11 & LOC649925 & NA & & \\
\hline & & & & rs7444 & 22 & $U B E 2 L 3$ & NA & & \\
\hline \multirow{8}{*}{ CLL-risk } & 2001 & European & $101 / 157$ & - & 6 & $\begin{array}{l}H L A-D R B 4 \\
\quad{ }^{*} 0103\end{array}$ & 2.74 & $2.5 \times 10^{-3}$ & {$[60]$} \\
\hline & \multirow{7}{*}{2008} & \multirow{7}{*}{ European } & \multirow{7}{*}{$1529 / 3115$} & rs17483466 & $2 q 13$ & $\begin{array}{l}\text { ACOXL, } \\
\text { BCL2L11 }\end{array}$ & $1.39(1.25-1.53)$ & $2.36 \times 10^{-10}$ & \multirow{7}{*}{ [55] } \\
\hline & & & & rs13397985 & $2 \mathrm{q} 37.1$ & SP140 * SP110* & $1.41(1.26-1.57)$ & $5.40 \times 10^{-10}$ & \\
\hline & & & & rs872071 & $6 \mathrm{p} 25.3$ & IRF4 & $1.54(1.41-1.69)$ & $1.91 \times 10^{-20}$ & \\
\hline & & & & rs9378805 & $6 \mathrm{p} 25.3$ & IRF4 & $1.51(1.38-1.65)$ & $4.62 \times 10^{-19}$ & \\
\hline & & & & rs735665 & 11q24.1 & GRAMD1B & $1.45(1.31-1.61)$ & $3.78 \times 10^{-12}$ & \\
\hline & & & & rs7176508 & $15 q 23$ & - & $1.37(1.26-1.50)$ & $4.54 \times 10^{-12}$ & \\
\hline & & & & rs11083846 & $19 q 13.32$ & PRKD2 & $1.35(1.22-1.49)$ & $3.96 \times 10^{-9}$ & \\
\hline
\end{tabular}


Table 1. Cont.

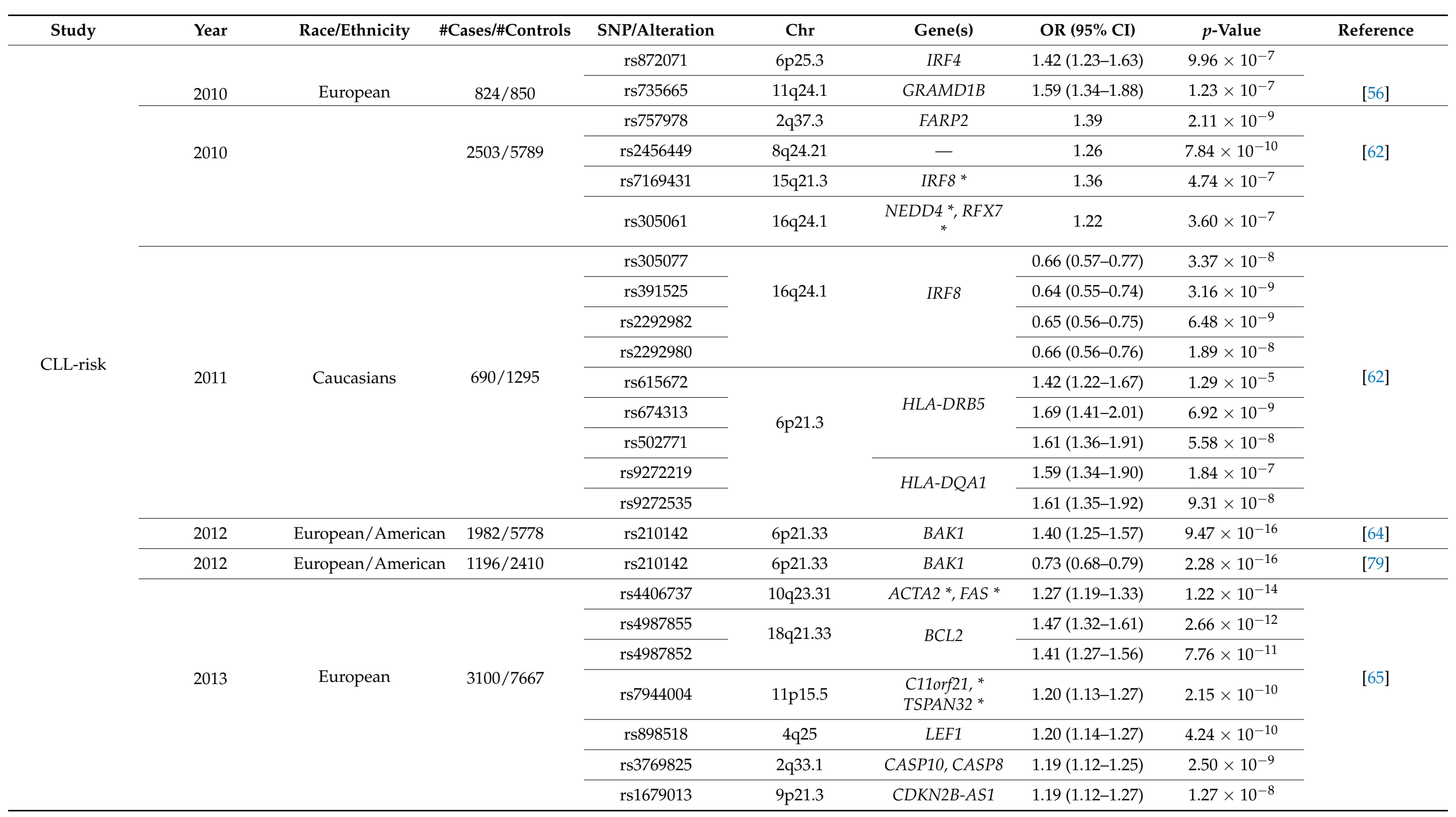


Table 1. Cont.

\begin{tabular}{|c|c|c|c|c|c|c|c|c|c|}
\hline Study & Year & Race/Ethnicity & \#Cases/\#Controls & SNP/Alteration & Chr & Gene(s) & OR $(95 \% \mathrm{CI})$ & $p$-Value & Reference \\
\hline \multirow{21}{*}{ CLL-risk } & \multirow{3}{*}{2013} & \multirow{3}{*}{ European } & \multirow{3}{*}{$3100 / 7667$} & rs4368253 & $18 \mathrm{q} 21.32$ & PMAIP1 & $1.19(1.12-1.27)$ & $2.51 \times 10^{-8}$ & \multirow{3}{*}[65]{} \\
\hline & & & & rs8024033 & $15 q 15.1$ & $B M F$ & $1.22(1.15-1.30)$ & $2.71 \times 10^{-10}$ & \\
\hline & & & & rs13401811 & $2 q 13$ & $\begin{array}{l}A C O X L^{*} \\
B C L 2 L 11 \text { * }\end{array}$ & $1.41(1.30-1.52)$ & $2.08 \times 10^{-18}$ & \\
\hline & \multirow{9}{*}{2014} & \multirow{9}{*}{ Europeans } & \multirow{9}{*}{$2883 / 8350$} & rs2236256 & $6 \mathrm{q} 25.2$ & IPCEF1 & $1.23(1.15-1.30)$ & $1.5 \times 10^{-10}$ & \multirow{9}{*}{ [66] } \\
\hline & & & & rs10936599 & $3 q 26.2$ & MYNN & $1.26(1.17-1.35)$ & $1.74 \times 10^{-9}$ & \\
\hline & & & & rs6858698 & $4 q 26$ & CAMK2D & $1.31(1.20-1.44)$ & $3.07 \times 10^{-9}$ & \\
\hline & & & & rs17246404 & 7q31.33 & POT1 & $1.22(1.14-1.31)$ & $3.40 \times 10^{-8}$ & \\
\hline & & & & rs1439287 & $2 q 13$ & ACOXL & 1.37 & $5 \times 10^{-15}$ & \\
\hline & & & & rs13397985 & $2 \mathrm{q} 37.1$ & SP140 & 1.43 & $5 \times 10^{-13}$ & \\
\hline & & & & rs735665 & $11 q 24.1$ & GRAMD1B & 1.64 & $4 \times 10^{-24}$ & \\
\hline & & & & rs7176508 & $15 q 23$ & DRAIC & 1.42 & $8 \times 10^{-18}$ & \\
\hline & & & & rs1044873 & $16 . q 24.1$ & IRF8 & 1.29 & $1 \times 10^{-9}$ & \\
\hline & \multirow{3}{*}{2014} & Caucasian & $3616 / 50,000$ & & 6 & \multirow{3}{*}{ 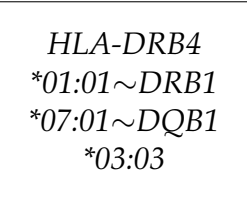 } & 1.49 & $1.79 \times 10^{-7}$ & \multirow{3}{*}[60]{} \\
\hline & & $\begin{array}{c}\text { African- } \\
\text { American }\end{array}$ & $413 / 50,000$ & - & 6 & & 28.03 & $2 \times 10^{-16}$ & \\
\hline & & Hispanic & $97 / 50,000$ & & 6 & & 13.86 & $9.59 \times 10^{-9}$ & \\
\hline & \multirow{6}{*}{2016} & \multirow{6}{*}{ Europeans } & \multirow{6}{*}{$5058 / 13,197$} & rs9880772 & $3 p 24.1$ & EOMES & $1.19(1.13-1.25)$ & $2.5 \times 10^{-11}$ & \multirow{6}{*}[67]{} \\
\hline & & & & rs73718779 & $6 \mathrm{p} 25.2$ & SERPINB6 & $1.26(1.16-1.36)$ & $1.97 \times 10^{-8}$ & \\
\hline & & & & rs9815073 & $3 q 28$ & $L P P$ & $1.18(1.11-1.25)$ & $3.26 \times 10^{-8}$ & \\
\hline & & & & rs9308731 & $2 q 13$ & BCL2L11 & $1.19(1.13-1.26)$ & $1 \times 10{ }^{11}$ & \\
\hline & & & & rs10028805 & $4 q 24$ & BANK1 & $1.16(1.10-1.22)$ & $7.19 \times 10^{-8}$ & \\
\hline & & & & rs1274963 & $3 p 22.2$ & CSRNP1 & $1.18(1.11-1.25)$ & $2.12 \times 10^{-7}$ & \\
\hline
\end{tabular}


Table 1. Cont.

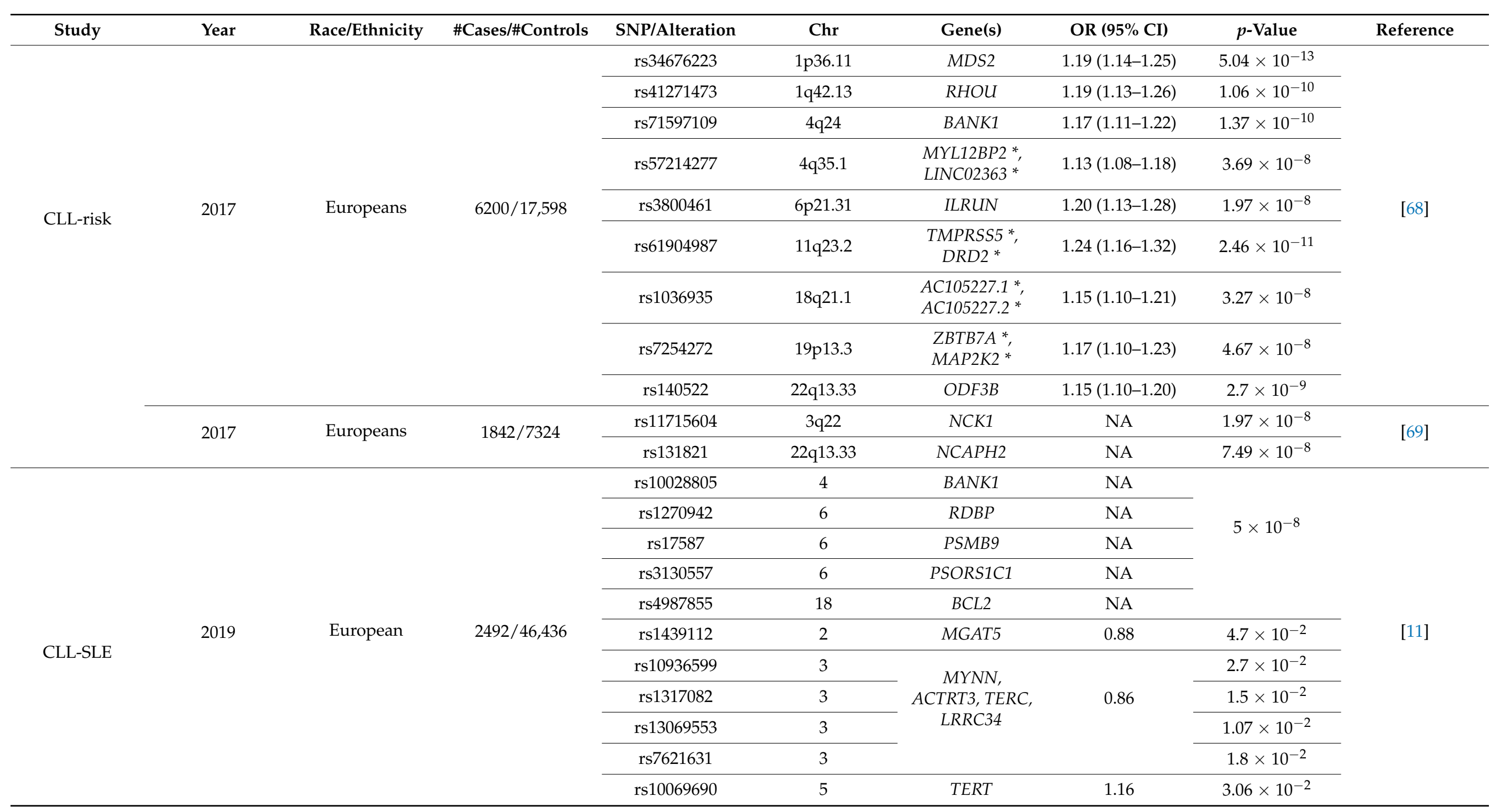


Table 1. Cont.

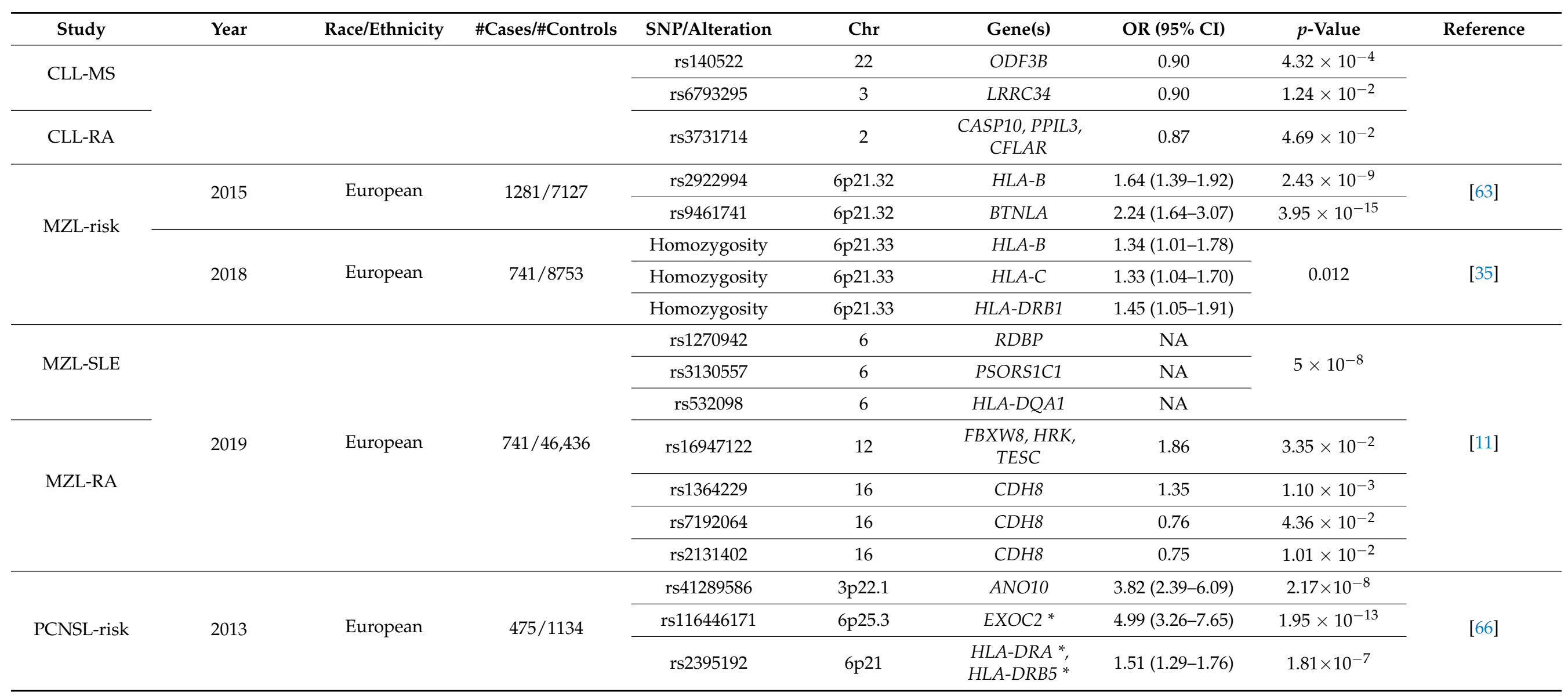

* Closest related gene; SLE: lupus erythematosus; MS: multiple sclerosis; RA: rheumatoid arthritis; NA: not available. 
Table 2. Associations of different loci by GWAS with survival for DLBCL and FL.

\begin{tabular}{|c|c|c|c|c|c|c|c|c|c|c|}
\hline Study & Year & Race/Ethnicity & \# Cases & SNP/Alteration & Chr & Gene(s) & HR (95\% CI) & $p$-Value & Outcome & Reference \\
\hline \multirow{5}{*}{ DLBCL } & \multirow{4}{*}{2015} & \multirow{4}{*}{ European } & \multirow{4}{*}{1537} & \multirow{2}{*}{ rs7712513 } & \multirow{2}{*}{$5 q 23.2$} & \multirow{2}{*}{$\begin{array}{c}\text { SNX2 * } \\
\text { SNCAIP * }\end{array}$} & $\begin{array}{c}1.49 \\
(1.29-1.72)\end{array}$ & $3.53 \times 10^{-8}$ & $\downarrow$ OS & \multirow{4}{*}{ [28] } \\
\hline & & & & & & & $\begin{array}{c}1.39 \\
(1.23-1.57) \\
\end{array}$ & $2.08 \times 10^{-7}$ & $\downarrow$ PFS & \\
\hline & & & & \multirow[b]{2}{*}{ rs7765004 } & \multirow{2}{*}{$6 q 21$} & \multirow{2}{*}{$\begin{array}{l}\text { MARCK * } \\
\text { HDACS2 * }\end{array}$} & $\begin{array}{c}1.47 \\
(1.27-1.71) \\
\end{array}$ & $5.36 \times 10^{-7}$ & $\downarrow$ OS & \\
\hline & & & & & & & $\begin{array}{c}1.38 \\
(1.22-1.57) \\
\end{array}$ & $7.09 \times 10^{-7}$ & $\downarrow$ PFS & \\
\hline & 2018 & European & 210 & - & $1 \mathrm{q} 23.3$ & FCGR2B & 2.18 & $5.7 \times 10^{-3}$ & $\downarrow$ OS & [40] \\
\hline \multirow{3}{*}{ FL } & \multirow{3}{*}{2014} & \multirow{3}{*}{ European } & \multirow{3}{*}{586} & rs10491178 & $17 q 24$ & $\begin{array}{l}A B C A 10^{*} \\
A B C A 6^{*}\end{array}$ & $\begin{array}{c}3.17 \\
(2.09-4.79)\end{array}$ & $5.24 \times 10^{-8}$ & $\downarrow \mathrm{PFS}$ & \multirow{3}{*}{ [47] } \\
\hline & & & & rs2466571 & $1 \mathrm{q} 32.2$ & $C D 46$ & $\begin{array}{c}0.73 \\
(0.58-0.91) \\
\end{array}$ & $6 \times 10^{-3}$ & $\uparrow \mathrm{EFS}$ & \\
\hline & & & & rs4073 & $4 q 13.3$ & IL8 & $\begin{array}{c}0.78 \\
(0.62-0.97)\end{array}$ & 0.02 & $\uparrow \mathrm{EFS}$ & \\
\hline
\end{tabular}

*Closest related gene; OS: overall survival; PFS: progression free survival; $\downarrow$ : inferior; EFS: event free survival; $\uparrow:$ superior. 
A two-stage study with $238 \mathrm{FL}$ cases and 1233 controls from United States found a variant in TAP2 gene (rs241447) to be associated with increase FL risk $(\mathrm{OR}=1.82 ; 95 \%$ $\mathrm{CI}=1.46-2.26 ; p=6.9 \times 10^{-8}$ ) but also with DLBCL (189 cases) risk, though DLBCL being not at genome-wide significance. TAP2 is part of the multidrug resistance protein (MRP)/TAP subfamily of ATP-binding cassette $(\mathrm{ABC})$ transporter, having an essential role for HLA class I protein loading on the cell surface and it is said that down-regulation or loss of function allows tumors to escape immune recognition [52]. One variant (rs6457327) near the psoriasis susceptibility locus (PSORS1) was found to be significantly associated to higher FL risk $\left(\mathrm{OR}=1.69 ; 5 \% \mathrm{CI}=1.43-2.00 ; p=4.7 \times 10^{-11}\right)$ among Europeans [55]. Skibola et al. recompiled information from 22 studies (4523 cases and 13,344 controls) from European populations and found five significant associated loci: $r \mathrm{~s} 6444305(\mathrm{OR}=1.21 ; 95 \%$ $\mathrm{CI}=1.14-1.28 ; p=1.10 \times 10^{-10}$ ) located in LPP which encodes a LIM domain containing protein that has cell adhesion, migration and proliferation roles and also found $836.4 \mathrm{~kb}$ upstream of $B C L 6$; rs13254990 $\left(\mathrm{OR}=1.18 ; 95 \% \mathrm{CI}=1.11-1.24 ; p=1.06 \times 10^{-8}\right)$ located intronic to $P V T 1$, a frequent translocation site in aggressive B-cell lymphomas; rs4938573 $\left(\mathrm{OR}=1.34 ; 95 \% \mathrm{CI}=1.26-1.46 ; p=5.79 \times 10^{-20}\right)$ located $12.6 \mathrm{~kb}$ upstream $C X C R 5$, involved in B-cell migration; rs4937362 (OR $\left.=1.19 ; 95 \% \mathrm{CI}=1.13-1.25 ; p=6.76 \times 10^{-11}\right)$ located near ETS1, a transcription factor for B-cell differentiation; rs17749561 (OR $=1.34 ; 95 \%$ $C I=1.22-1.47 ; p=8.28 \times 10^{-10}$ ) located near BCL2, an anti-apoptotic oncogene. Furthermore, another interesting, though not genome-wide significant, rs2681416 variant (near CD86) showed increased risk of FL [54]. A Chinese study evaluating 792 cases and 1542 controls used additive genetic models adjusted with the false-positive rate probability to evaluate GWAS significance. This study found a variant on IRF4 (rs872071), a crucial gene for B-cell development, to be associated with increased FL risk [51]. Moreover, this variant was found to be also associated with CLL risk in two additional European population studies [56,57]. Additionally, five more variants (CFB, MSH5, TNXB, LOC649925, and UBE2L3) on chromosome 6 were associated with FL and SLE risk [11].

$\mathrm{ABC}$ transporter variants associated with FL with worse PFS are $A B C A 10$ and $A B C A 6$ $\left(\mathrm{rs} 10491178 ; \mathrm{HR}=3.17 ; 95 \% \mathrm{CI}=2.09-4.79 ; p=5.24 \times 10^{-8}\right)$ which is in high LD $(\mathrm{r} 2>0.8)$ with another variant within the binding site of a transcription factor, PAX5, that has been correlated with aggressive subsets of B-cell NHL. These results were found among Europeans along with a variant on $C D 46$ (rs2466571; $\mathrm{HR}=0.73 ; 95 \% \mathrm{CI}=0.58-0.91$; $\left.p=6 \times 10^{-3}\right), I L 8(\mathrm{rs} 4073 ; \mathrm{HR}=0.78 ; 95 \% \mathrm{CI}=0.62-0.97 ; p=0.02)$, and MTHFR (rs1801131; $\mathrm{HR}=0.59 ; 95 \% \mathrm{CI}=0.45-0.77 ; p=1 \times 10^{-4}$ ), albeit positively associated with event-free survival after adjusting for age, sex and population stratification. [47].

\section{3. $C L L$}

Another indolent lymphoma is CLL since the five-year survival rate is $\sim 85 \%$ and it is characterized by a very rare incidence among Asian descendants compared to Caucasians and nearly double in males compared to females. Risk factors with CLL were previously identified to be family history of NHL ( $\mathrm{OR}=1.92 ; 95 \% \mathrm{CI}=1.42$ to 2.61$)$, hepatitis $\mathrm{C}$ virus infection ( $\mathrm{OR}=2.08 ; 95 \% \mathrm{CI}=1.23$ to 3.49$)$, and height $(\mathrm{OR}=1.08,95 \% \mathrm{CI}=1.00-1.17$, $p=0.049)$ showing a slightly stronger trend among women (OR $=1.15,95 \%$ CI: $1.01-1.31$, $p=0.036)$. Conversely, immune function through allergy had a protective effect $(\mathrm{OR}=0.87$; $95 \% \mathrm{CI}=0.77$ to 0.98 ) [10,58]. Additionally, an analysis of 13 cancer types including 49,492 cancer case patients and 34,131 control patients found that individuals with a high risk score for CLL were at an increased relative risk of DLBCL ( $R R=1.12,95 \% \mathrm{CI}=1.07$ to 1.16) [59]. HLA associations to CLL were mainly reported for the expanded haplotype DRB4*01:01 DRB1*07:01 DQB1*03:03 in Caucasians (OR $=1.49 ; p=1.79 \times 10^{-7}$ ), African Americans $\left(\mathrm{OR}=28.03 ; p=2 \times 10^{-16}\right)$, and Hispanics $\left(\mathrm{OR}=13.86 ; p=9.59 \times 10^{-9}\right)$ and HLA-DRB4*0103 in a German study ( $R R=2.74 ; p=0.0025)[60,61]$. Other study from Caucasian population, found five variants from which two were associated with increase disease risk at genome-wide significance, one located near HLA-DRB5 (rs674317) and the 
other near HLA-DQA1 (rs9272535) [62]. On the other hand, so far there are no indicators of HLA zygosity associations with CLL risk $[35,63]$.

Because there have been several studies accessing CLL risk outside the HLA region, some variants have been validated across GWAS, for example variant rs17483466 (2q13; ACOXL and BCL2L11; $\left.\mathrm{p}_{1}=2.36 \times 10^{-10} ; \mathrm{p}_{2}=5 \times 10^{-9} ; \mathrm{p}_{3}=4 \times 10^{-17}\right)$, rs735665 (11q24.1; GRAMD1B; $\left.\mathrm{p}_{1}=3.78 \times 10^{-12} ; \mathrm{p}_{2}=4 \times 10^{-24}\right)$ and $\mathrm{rs} 210142\left(6 \mathrm{p} 21.31 ; B A K 1 ; \mathrm{p}_{1}=9.47 \times 10^{-16}\right.$; $\left.\mathrm{p}_{2}=2.28 \times 10^{-16}\right)[56,64-66]$. Some biologically interesting variants include one encoding a protein involved in the signal transduction downstream of Ras, PCEF1, which happens to reside within a strong enhancer element ( $\mathrm{rs} 2236256$; $\left.\mathrm{OR}=1.23 ; p=1.5 \times 10^{-10}\right)$; a telomer protecting protein, POT1 ( $\left.\mathrm{rs} 17246404 ; \mathrm{OR}=1.22 ; p=3.40 \times 10^{-8}\right)$ [66]; a member of the tumor necrosis factor which is essential for the signaling cascade in apoptosis, ACTA2/FAS (rs4406737; $\left.\mathrm{OR}=1.27 ; p=1.22 \times 10^{-14}\right)$; a lymphocyte's apoptosis blocker, BCL2 (rs4987855; OR = 1.47; $p=2.66 \times 10^{-12}$ ) [65]; a member of the T-box gene family that regulates $\mathrm{CD} 8^{+} \mathrm{T}$-cell differentiation and immunity, EOMES (rs9880772; $\mathrm{p}_{1}=3 \times 10^{-11} ; \mathrm{p}_{2}=\times 10^{-9} ; \mathrm{p}_{3}=2 \times 10^{-9}$ ), which is also critical during Fas deficiency for lymphoproliferation [67-69]; a B-cell specific scaffold protein involved in B-cell antigen receptors, BANK1 (rs71597109; $\mathrm{OR}=1.17 ; p=1.37 \times 10^{-10}$ ); a master regulator of lymphocyte fate (B-cell vs T-cell) which is also involved in NOTCH pathway activation, ZBTB7A $\left(\right.$ rs7254272; OR $\left.=1.17 ; p=4.67 \times 10^{-8}\right)$ [68] and a regulator of the PI3K/Akt pathway, NCK1 (rs11715604; $p=1.97 \times 10^{-8}$ ) [69].

Other implicated loci by GWAS include: 2q33.1 (rs3769825; CASP10/CASP8; $p=2.5 \times 10^{-9}$ ), 2q37.1 (rs13397985; SP140; $\left.p=5.40 \times 10^{-10}\right), 2 \mathrm{q} 37.3\left(\mathrm{rs} 757978 ; \mathrm{FARP} 2 ; \mathrm{OR}=1.39 ; p=2.11 \times 10^{-9}\right)$, 3q25.2 (rs10936599; MYNN; $\left.p=1.74 \times 10^{-9}\right), 4 \mathrm{q} 25\left(\mathrm{rs} 898518 ; L E F 1 ; p=4.24 \times 10^{-10}\right), 4 \mathrm{q} 26$ $\left(\mathrm{rs} 6858698 ; C A M K 2 D ; p=3.07 \times 10^{-9}\right), 6 \mathrm{p} 25.3(\mathrm{rs} 872071$ and rs9378805; IRF4; $p=1.91 \times$ $\left.10^{-20}\right), 8 \mathrm{q} 24.21\left(\mathrm{rs} 2456449 ; p=7.84 \times 10^{-10}\right), 11 \mathrm{q} 24.1\left(\mathrm{rs} 735665 ; G R A M D 1 B ; p=3.78 \times 10^{-12}\right)$, 12q24.13 (rs10735079; OAS3; $\left.p=2.34 \times 10^{-8}\right), 15 \mathrm{q} 23\left(\mathrm{rs} 7176508 ;\right.$ DRAIC; $\left.p=8 \times 10^{-18}\right), 15 \mathrm{q} 21.3$ $\left(\right.$ rs7169431; IRF8; $\left.p=4.74 \times 10^{-7}\right), 15 q 23\left(\right.$ rs7176508; $\left.p=4.54 \times 10^{-12}\right), 16 \mathrm{q} 24.1(\mathrm{rs} 305061 ; N E D D 4$ and RFX7; $\left.p=3.60 \times 10^{-7}\right)$, and 19q13.32 (rs11083846; PRKD2; $\left.p=3.96 \times 10^{-9}\right)[56,64,65,70,71]$. Slager et al. found four additional IRF8 variants associated to both decreased CLL risk and increased IRF8 expression (using lymphocytes cell lines data) which is opposite to the previous finding by Crowther et al. one year earlier [57,62]. IRF4 and IRF8 are a strong finding due to its role as key regulator of B-cell development, proliferation, and lymphogenesis; furthermore, associations for variant (rs872071) were also found for FL [51,70].

In spite of these findings several CLL associated risk variants have been also found for SLE, MS, and RA, only few have reached genome-wide significance; for example, a variant on gene BCL2 (rs4987855) which has anti-apoptotic activity [11].

\subsection{MZL}

Marginal zone lymphoma, which comprises $10 \%$ of NHL cases, originate from marginal zone B cells present as three different types: extranodal MZL of mucosa-associated lymphoid tissue (EMZL) and splenic MZL (SMZL) and nodal MZL (NMZL) [64,65,72,73]. Risk factors for MZL include autoimmune conditions (EMZL OR $=6.40,95 \% \mathrm{CI}=4.24-9.68$; NMZL OR $=7.80,95 \%$ CI $=3.32-18.33$; SMZL OR $=4.25,95 \%$ CI $=1.49-12.14$ ), hepatitis $C$ virus seropositivity (EMZL OR $=5.29,95 \% C I=2.48-11.28$ ), self-reported peptic ulcers (EMZL OR $=1.83,95 \% \mathrm{CI}=1.35-2.49$ ), or family history NHL (NMZL OR $=2.82$, $95 \% \mathrm{CI}=1.33-5.98)$. On the contrary, triglycerides levels were found as protective factor $(\mathrm{OR}=0.90 ; 95 \%$ CI, 0.83-0.99) [18,66,74]. GWAS studies in MZL, which are less extensive. One study comprised 1281 cases and 7127 controls of European ancestry in which a variant in HLA-B allele (rs2922994; OR $=1.64 ; 95 \% \mathrm{CI}=1.39-1.92 ; p=2.43 \times 10^{-9}$ ) was found to be associated with increased MZL risk taken together with a variant on BTNL2 (rs9461741; $\mathrm{OR}=2.24,95 \% \mathrm{CI}=1.64-3.07 ; p=3.95 \times 10^{-15}$ ), a gene involved lymphocyte activation and antigen presentation [64,72]. A second study assessing the role of HLA homozygosity in MZL risk (increased for HLA-B, HLA-C, and HLA-DRB1). A third study assessed the pleiotropy with SLE (RDBP, PSORS1C1, and HLA-DQA1) and RA (CDH8) [11]. 


\subsection{PCNSL}

Recognized as mature post-germinal B cells (ABC subtype like), the central nervous system (CNS) DLBCL represents only $\leq 1 \%$ of all lymphomas and approximately $2 \%$ of all primary CNS tumors; furthermore, $95 \%$ of tumors have a comparative histology with systemic DLBCL $[67,75]$. Since the blood brain barrier impedes R-CHOP treatment, high dose methotrexate (HD-MTX, $>3 \mathrm{~g} / \mathrm{m}^{2}$ ) based regimens are the gold standard for PCNSL patients resulting in a five year survival rate of $30 \%$ and high risk of clinical neurotoxicity specially in patients $>60[68,76]$. Furthermore, PCNSL is less frequently associated with any atopic disorder ( $\mathrm{OR}=0.54,95 \% \mathrm{CI}=0.33$ to 0.87$)$, but it is strongly associated with a family history of NHL (OR $=4.11,95 \% \mathrm{CI}=1.58$ to 10.66$)$ and less clearly with lifetime cigarette exposure ( $\mathrm{OR}=1.51,95 \% \mathrm{CI}=0.83$ to 2.74 , for $1-10$ pack-years vs. nonsmoker) [30]. The only study that has reported associations between genetic variants and PCNSL risk, evaluated 475 cases and 1134 controls from French population. This study found one variant at loci 6p25.3 (rs116446171; EXOC2; $p=1.95 \times 10^{-13}$ ) previously found to be associated with DLBCL risk, other at loci 3p22.1 (rs41289586, ANO10, $p=2.17 \times 10^{-8}$ ) and one strongly associated at HLA allele (rs2395192; between HLA-DRA and HLADRB5; $p=1.81 \times 10^{-7}$ ). ANO10 is a calcium-activated chloride channel transmembrane protein that might be involved in the innate immune defense and indirect activation of the Ras/Raf/MEK/ERK signaling pathway which affects cell proliferation $[67,69,75,77]$.

\section{Conclusions}

Initially data suggested increased risk of any DLBCL, FL, CLL, MZL, or PCNSL if family history of NHL was present, though specific genetic attributions for specific risk or prognosis had been lacking [30,49,58,74]. The decreasing cost and bioinformatics limitations for NGS and GWAS have augmented the ability to detect the genetic risk for NHL etiologies, pleiotropy with other diseases and, more importantly, clinical applications. Despite that many HLA alleles have been found to be not only associated to specific lymphoma subtypes but also within subtypes (DLBCL and FL) or with autoimmune diseases (MS, SLE, or RA), today they remain just informative for prognosis since no clinical use has been made $[7,11,14]$. Peptide diversity reduction can increase tumoral escape from immune surveillance, which can be partially a consequence of HLA homozygosity, which was found to be a risk factor for most of the reviewed lymphomas [35,63].

On the other hand, GWAS findings outside HLA loci have led to the discovery of B-cell NHLs shared genetic risk with autoimmune diseases leading to finding of genes involved in cell cycle, apoptosis and telomere length, though studies are limited and total risk is still modest at genome wide-significance.

Taken together that hepatitis seropositivity is associated with DLBCL, CLL and MZL risk and that AICDA promiscuous off-target activity, highly present in lymphomas and induced by viral infection, can provoke important alterations (example translocations of PD-L1/PD-L2 with PIM1, TP63, and IGH loci). Further efforts should be made to find correlations between these variables [41,80-82]. Future efforts should also be directed to extend studies among non-Caucasian populations, in order to clarify differences in susceptibility variants, and among B-cell NHLs subtypes since most studies have focused on DLBCL. Furthermore, there is an unmet need to translate theoretical information into clinical practice which has been done, for example, with the use of adavosertib, an WEE1 inhibitor, to increase response in DLBCL patients. In line with this, the incorporation of single-cell sequencing technology can help identify B-cell stages (dark/light zone) and cell cycle phases to further amplify the possibilities for therapy options $[17,46,83]$.

Author Contributions: Conceptualization, I.H.-V., K.H.-X., and A.A.; methodology, I.H.-V., and M.B.; resources, I.H.-V., and K.L.; data curation, I.H.-V., K.H.-X., K.M., and A.A.; writing-original draft preparation, I.H.-V., K.L., and M.B.; writing-review and editing, all authors; visualization, all authors; supervision, I.H.-V., K.H.-X., and A.A; project administration, I.H.-V. and A.A.; funding acquisition, K.H.-X. and A.A. All authors have read and agreed to the published version of the manuscript. 
Funding: This work was partially funded by the P DGOS/INCa PRT-K grant 2017-1-RT-04. A.A has also been supported by French Agence Nationale de la Recherche, as part of the second Investissements d'Avenir program (ANR-18-RHUS-0012) and by RAM Active Investments. I.H.-V. is supported by l'Association pour la Recherche sur les Tumeurs Cérébrales (ARTC).

Conflicts of Interest: The authors declare no conflict of interest.

$\begin{array}{ll}\text { Abbreviations } & \\ \text { NHL } & \text { Non-Hodgkin's lymphoma } \\ \text { HL } & \text { Hodgkin's lymphoma } \\ \text { DLBCL } & \text { Diffuse large B-cell lymphoma } \\ \text { FL } & \text { Follicular lymphoma } \\ \text { CLL } & \text { Chronic lymphocytic leukemia } \\ \text { MZL } & \text { Marginal zone lymphoma } \\ \text { PCNSL } & \text { Primary central nervous system lymphoma } \\ \text { NGS } & \text { Next generation sequencing } \\ \text { GWAS } & \text { Genome-wide association studies } \\ \text { HLA } & \text { Human leukocyte antigen } \\ \text { MHC } & \text { Major histocompatibility complex } \\ \text { NK } & \text { Natural killer } \\ \text { R-CHOP } & \text { Rituximab combined with cyclophosphamide, doxorubicin, vincristine, } \\ \text { GCB } & \text { and prednisolone treatment } \\ \text { ABC } & \text { Germinal center B-cell } \\ \text { OR } & \text { Activated B-cell } \\ \text { CI } & \text { Odds ratio } \\ \text { LD } & \text { Confidence interval } \\ \text { SLE } & \text { Linkage disequilibrium } \\ \text { MS } & \text { Lupus erythematosus } \\ \text { RA } & \text { Multiple sclerosis } \\ \text { AICDA } & \text { Rheumatoid arthritis } \\ \text { GEO } & \text { Activation-induced cytidine deaminase } \\ \text { ABC } & \text { Genome Expression Omnibus } \\ \text { EMZL } & \text { ATP-binding cassette } \\ \text { HD-MTX } & \text { Extranodal MZL of mucosa-associated lymphoid tissue } \\ & \text { High dose methotrexate }\end{array}$

\section{References}

1. Al-Naeeb, A.B.; Ajithkumar, T.; Behan, S.; Hodson, D. Non-Hodgkin lymphoma. BMJ 2018, 362, k3204. [CrossRef] [PubMed]

2. Hoang-Xuan, K.; Bessell, E.; Bromberg, J.E.C.; Hottinger, A.F.; Preusser, M.; Rudà, R.; Schlegel, U.; Siegal, T.; Soussain, C.; Abacioglu, U.; et al. Diagnosis and treatment of primary CNS lymphoma in immunocompetent patients: Guidelines from the European Association for Neuro-Oncology. Lancet Oncol. 2015, 16, e322-e332. [CrossRef]

3. Smedby, K.E. Epidemiology and etiology of non-Hodgkin lymphoma-A review. Acta Oncol. 2006, 45, 258-271. [CrossRef] [PubMed]

4. Parihar, A.S.; Singh, R.; Shaik, S.; Negi, B.S.; Rajguru, J.P.; Patil, P.B.; Sharma, U. Non-Hodgkin's lymphoma: A review. J. Fam. Med. Prim. Care 2020, 9, 1834-1840. [CrossRef] [PubMed]

5. Swerdlow, S.H.; Campo, E.; Pileri, S.A.; Harris, N.L.; Stein, H.; Siebert, R.; Advani, R.; Ghielmini, M.; Salles, G.A.; Zelenetz, A.D.; et al. The 2016 revision of the World Health Organization classification of lymphoid neoplasms. Blood 2016, 127, 2375-2390. [CrossRef] [PubMed]

6. Braggio, E.; Van Wier, S.; Ojha, J.; McPhail, E.; Asmann, Y.W.; Egan, J.; Da Silva, J.A.; Schiff, D.; Lopes, M.B.; Decker, P.A.; et al. Genome-Wide Analysis Uncovers Novel Recurrent Alterations in Primary Central Nervous System Lymphomas. Clin. Cancer Res. 2015, 21, 3986-3994. [CrossRef] [PubMed]

7. Zhong, C.; Cozen, W.; Bolanos, R.; Song, J.; Wang, S.S. The role ofHLAvariation in lymphoma aetiology and survival. J. Intern. Med. 2019, 286, 154-180. [CrossRef] [PubMed]

8. Olszewski, A.J.; Castillo, J.J. Survival of patients with marginal zone lymphoma. Cancer 2012, 119, 629-638. [CrossRef]

9. Morton, L.M.; Slager, S.L.; Cerhan, J.R.; Wang, S.S.; Vajdic, C.M.; Skibola, C.F.; Bracci, P.M.; De Sanjosé, S.; Smedby, K.E.; Chiu, B.C.H.; et al. Etiologic Heterogeneity Among Non-Hodgkin Lymphoma Subtypes: The InterLymph Non-Hodgkin Lymphoma Subtypes Project. J. Nat. Cancer Inst. Monogr. 2014, 2014, 130-144. [CrossRef]

10. Moore, A.; Kane, E.; Wang, Z.; Panagiotou, O.A.; Teras, L.R.; Monnereau, A.; Doo, N.W.; Machiela, M.J.; Skibola, C.F.; Slager, S.L.; et al. Genetically Determined Height and Risk of Non-hodgkin Lymphoma. Front. Oncol. 2020, 9, 1539. [CrossRef] 
11. Din, L.; Sheikh, M.; Kosaraju, N.; Smedby, K.E.; Bernatsky, S.; Berndt, S.I.; Skibola, C.F.; Nieters, A.; Wang, S.; McKay, J.D.; et al. Genetic overlap between autoimmune diseases and non-Hodgkin lymphoma subtypes. Genet. Epidemiol. 2019, 43, 844-863. [CrossRef]

12. Smedby, K.E.; Ponzoni, M. The aetiology of B-cell lymphoid malignancies with a focus on chronic inflammation and infections. J. Intern. Med. 2017, 282, 360-370. [CrossRef] [PubMed]

13. Hjalgrim, H.; Rostgaard, K.; Johnson, P.C.D.; Lake, A.; Shield, L.; Little, A.M.; Ekstrom-Smedby, K.; Adami, H.O.; Glimelius, B.; Hamilton-Dutoit, S.; et al. HLA-A alleles and infectious mononucleosis suggest a critical role for cytotoxic T-cell response in EBV-related Hodgkin lymphoma. Proc. Nat. Acad. Sci. USA 2010, 107, 6400-6405. [CrossRef]

14. Mills, M.C.; Rahal, C. A scientometric review of genome-wide association studies. Commun. Biol. 2019, 2, 9. [CrossRef] [PubMed]

15. Wang, M.; Xu, S. Statistical power in genome-wide association studies and quantitative trait locus mapping. Heredity 2019, 123, 287-306. [CrossRef]

16. Di Paolo, A.; Arrigoni, E.; Luci, G.; Cucchiara, F.; Danesi, R.; Galimberti, S. Precision Medicine in Lymphoma by Innovative Instrumental Platforms. Front. Oncol. 2019, 9, 1417. [CrossRef]

17. De Jong, M.R.W.; Visser, L.; Huls, G.; Diepstra, A.; Van Vugt, M.; Ammatuna, E.; Van Rijn, R.S.; Vellenga, E.; Berg, A.V.D.; Fehrmann, R.S.N.; et al. Identification of relevant drugable targets in diffuse large B-cell lymphoma using a genome-wide unbiased CD20 guilt-by association approach. PLoS ONE 2018, 13, e0193098. [CrossRef]

18. Kleinstern, G.; Camp, N.J.; Berndt, S.I.; Birmann, B.M.; Nieters, A.; Bracci, P.M.; McKay, J.D.; Ghesquières, H.; Lan, Q.; Hjalgrim, H.; et al. Lipid Trait Variants and the Risk of Non-Hodgkin Lymphoma Subtypes: A Mendelian Randomization Study. Cancer Epidemiol. Biomark. Prev. 2020, 29, 1074-1078. [CrossRef]

19. Bernatsky, S.; García, H.A.V.; Spinelli, J.J.; Gaffney, P.E.; Smedby, K.; Ramsey-Goldman, R.; Wang, S.S.; Adami, H.-O.; Albanes, D.; Angelucci, E.; et al. Lupus-related single nucleotide polymorphisms and risk of diffuse large B-cell lymphoma. Lupus Sci. Med. 2017, 4, e000187. [CrossRef] [PubMed]

20. Trowsdale, J.; Knight, J.C. Major Histocompatibility Complex Genomics and Human Disease. Annu. Rev. Genom. Hum. Genet. 2013, 14, 301-323. [CrossRef] [PubMed]

21. Amiel, J. Study of the Leukocyte Phenotypes in Hodgkin's Disease. In Histocompatibility Testing; Munksgaard: Copenhagen, Denmark, 1967; pp. 79-81.

22. Sanchez-Mazas, A. A review of HLA allele and SNP associations with highly prevalent infectious diseases in human populations. Swiss Med. Wkly. 2020, 150. [CrossRef] [PubMed]

23. Dendrou, C.A.; Petersen, J.; Rossjohn, J.; Fugger, L. HLA variation and disease. Nat. Rev. Immunol. 2018, 18, 325-339. [CrossRef] [PubMed]

24. Deakin, J.E.; Papenfuss, A.T.; Belov, K.; Cross, J.G.R.; Coggill, P.; Palmer, S.; Sims, S.; Speed, T.P.; Beck, S.; Graves, J.A.M. Evolution and comparative analysis of the MHC Class III inflammatory region. BMC Genom. 2006, 7, 281. [CrossRef] [PubMed]

25. Robinson, J.; Soormally, A.R.; Hayhurst, J.D.; Marsh, S.G.E. The IPD-IMGT/HLA Database-New developments in reporting HLA variation. Hum. Immunol. 2016, 77, 233-237. [CrossRef]

26. Sewell, A.K. Why must T cells be cross-reactive? Nat. Rev. Immunol. 2012, 12, 669-677. [CrossRef]

27. Saunders, P.M.; Vivian, J.P.; O'Connor, G.M.; Sullivan, L.C.; Pymm, P.G.; Rossjohn, J.; Brooks, A.G. A bird's eye view of NK cell receptor interactions with their MHC class I ligands. Immunol. Rev. 2015, 267, 148-166. [CrossRef]

28. Ghesquieres, H.; Slager, S.L.; Jardin, F.; Veron, A.S.; Asmann, Y.W.; Maurer, M.J.; Fest, T.; Habermann, T.M.; Bene, M.C.; Novak, A.J.; et al. Genome-Wide Association Study of Event-Free Survival in Diffuse Large B-Cell Lymphoma Treated With Immunochemotherapy. J. Clin. Oncol. 2015, 33, 3930-3937. [CrossRef]

29. Wright, G.; Tan, B.; Rosenwald, A.; Hurt, E.H.; Wiestner, A.; Staudt, L.M. A gene expression-based method to diagnose clinically distinct subgroups of diffuse large B cell lymphoma. Proc. Nat. Acad. Sci. USA 2003, 100, 9991-9996. [CrossRef]

30. Cerhan, J.R.; Kricker, A.; Paltiel, O.; Flowers, C.R.; Wang, S.S.; Monnereau, A.; Blair, A.; Maso, L.D.; Kane, E.V.; Nieters, A.; et al. Medical History, Lifestyle, Family History, and Occupational Risk Factors for Diffuse Large B-Cell Lymphoma: The InterLymph Non-Hodgkin Lymphoma Subtypes Project. J. Nat. Cancer Inst. Monogr. 2014, 2014, 15-25. [CrossRef]

31. Park, H.Y.; Hong, Y.C.; Lee, K.; Koh, J. Vitamin D status and risk of non-Hodgkin lymphoma: An updated meta-analysis. PLoS ONE 2019, 14, e0216284. [CrossRef]

32. Cerhan, J.R.; Berndt, S.I.; Vijai, J.; Ghesquières, H.; McKay, J.D.; Wang, S.S.; Wang, Z.; Yeager, M.; Conde, L.; De Bakker, P.I.; et al. Genome-wide association study identifies multiple susceptibility loci for diffuse large B cell lymphoma. Nat. Genet. 2014, 46, 1233-1238. [CrossRef] [PubMed]

33. Bassig, B.A.; Cerhan, J.R.; Au, W.-Y.; Kim, H.N.; Sangrajrang, S.; Hu, W.; Tse, J.; Berndt, S.; Zheng, T.; Zhang, H.; et al. Genetic susceptibility to diffuse large B-cell lymphoma in a pooled study of three Eastern Asian populations. Eur. J. Haematol. 2015, 95, 442-448. [CrossRef] [PubMed]

34. Smedby, K.E.; Foo, J.N.; Skibola, C.F.; Darabi, H.; Conde, L.; Hjalgrim, H.; Kumar, V.; Chang, E.T.; Rothman, N.; Cerhan, J.R.; et al. GWAS of Follicular Lymphoma Reveals Allelic Heterogeneity at 6p21.32 and Suggests Shared Genetic Susceptibility with Diffuse Large B-cell Lymphoma. PLoS Genet. 2011, 7, e1001378. [CrossRef] [PubMed]

35. Wang, S.S.; Carrington, M.; Berndt, S.I.; Slager, S.L.; Bracci, P.M.; Voutsinas, J.; Cerhan, J.R.; Smedby, K.E.; Hjalgrim, H.; Vijai, J.; et al. HLA Class I and II Diversity Contributes to the Etiologic Heterogeneity of Non-Hodgkin Lymphoma Subtypes. Cancer Res. 2018, 78, 4086-4096. [CrossRef] [PubMed] 
36. Kleinstern, G.; Yan, H.; Hildebrandt, M.A.T.; Vijai, J.; Berndt, S.I.; Ghesquières, H.; McKay, J.; Wang, S.S.; Nieters, A.; Ye, Y.; et al. Inherited variants at 3q13.33 and 3p24.1 are associated with risk of diffuse large B-cell lymphoma and implicate immune pathways. Hum. Mol. Genet. 2019, 29, 70-79. [CrossRef]

37. Aslan, K.; Turco, V.; Blobner, J.; Sonner, J.K.; Liuzzi, A.R.; Núñez, N.G.; De Feo, D.; Kickingereder, P.; Fischer, M.; Green, E.; et al. Heterogeneity of response to immune checkpoint blockade in hypermutated experimental gliomas. Nat. Commun. 2020, 11, 931. [CrossRef]

38. Basso, K.; Dalla-Favera, R. BCL6. Adv. Immunol. 2010, 105, 193-210. [CrossRef]

39. Kumar, V.; Matsuo, K.; Takahashi, A.; Hosono, N.; Tsunoda, T.; Kamatani, N.; Kong, S.Y.; Nakagawa, H.; Cui, R.; Tanikawa, C.; et al. Common variants on 14q32 and 13q12 are associated with DLBCL susceptibility. J. Hum. Genet. 2011, 56, 436-439. [CrossRef]

40. Arthur, S.E.; Jiang, A.; Grande, B.M.; Alcaide, M.; Cojocaru, R.; Rushton, C.K.; Mottok, A.; Hilton, L.K.; Lat, P.K.; Zhao, E.Y.; et al. Genome-wide discovery of somatic regulatory variants in diffuse large B-cell lymphoma. Nat. Commun. 2018, 9, 4001. [CrossRef]

41. Georgiou, K.; Chen, L.; Berglund, M.; Ren, W.; De Miranda, N.F.C.C.; Lisboa, S.; Fangazio, M.; Zhu, S.; Hou, Y.; Wu, K.; et al. Genetic basis of PD-L1 overexpression in diffuse large B-cell lymphomas. Blood 2016, 127, 3026-3034. [CrossRef]

42. Muramatsu, M.; Kinoshita, K.; Fagarasan, S.; Yamada, S.; Shinkai, Y.; Honjo, T. Class Switch Recombination and Hypermutation Require Activation-Induced Cytidine Deaminase (AID), a Potential RNA Editing Enzyme. Cell 2000, 102, 553-563. [CrossRef]

43. Duclos, C.M.; Champagne, A.; Carrier, J.C.; Saucier, C.; Lavoie, C.L.; Denault, J.-B. Caspase-mediated proteolysis of the sorting nexin 2 disrupts retromer assembly and potentiates Met/hepatocyte growth factor receptor signaling. Cell Death Discov. 2017, 3, 16100. [CrossRef] [PubMed]

44. Li, Y.; Yu, Z.; Jiang, T.; Shao, L.; Liu, Y.; Li, N.; Wu, Y.; Zheng, C.; Wu, X.; Zhang, M.; et al. SNCA, a novel biomarker for Group 4 medulloblastomas, can inhibit tumor invasion and induce apoptosis. Cancer Sci. 2018, 109, 1263-1275. [CrossRef] [PubMed]

45. Fong, L.W.R.; Yang, D.C.; Chen, C.-H. Myristoylated alanine-rich C kinase substrate (MARCKS): A multirole signaling protein in cancers. Cancer Metastasis Rev. 2017, 36, 737-747. [CrossRef]

46. De Jong, M.R.W.; Langendonk, M.; Reitsma, B.; Herbers, P.; Nijland, M.; Huls, G.; Berg, A.V.D.; Ammatuna, E.; Visser, L.; Van Meerten, T. WEE1 Inhibition Enhances Anti-Apoptotic Dependency as a Result of Premature Mitotic Entry and DNA Damage. Cancers 2019, 11, 1743. [CrossRef]

47. Baecklund, F.; Foo, J.N.; Bracci, P.M.; Darabi, H.; Karlsson, R.; Hjalgrim, H.; Rosenquist, R.; Adami, H.-O.; Glimelius, U.I.B.; Melbye, M.; et al. A comprehensive evaluation of the role of genetic variation in follicular lymphoma survival. BMC Med. Genet. 2014, 15, 113. [CrossRef]

48. Skibola, C.F.; Akers, N.K.; Conde, L.; Ladner, M.; Hawbecker, S.K.; Cohen, F.; Ribas, F.; Erlich, H.A.; Goodridge, D.; Trachtenberg, E.A.; et al. Multi-locus HLA class I and II allele and haplotype associations with follicular lymphoma. Tissue Antigens 2012, 79, 279-286. [CrossRef]

49. Linet, M.S.; Vajdic, C.; Morton, L.M.; De Roos, A.J.; Skibola, C.F.; Boffetta, P.; Cerhan, J.R.; Flowers, C.R.; De Sanjosé, S.; Monnereau, A.; et al. Medical History, Lifestyle, Family History, and Occupational Risk Factors for Follicular Lymphoma: The InterLymph Non-Hodgkin Lymphoma Subtypes Project. J. Nat. Cancer Inst. Monogr. 2014, 2014, 26-40. [CrossRef]

50. Conde, L.; Halperin, E.; Akers, N.K.; Brown, K.M.; Smedby, K.E.; Rothman, N.; Nieters, A.; Slager, S.L.; Brooks-Wilson, A.; Agana, L.; et al. Genome-wide association study of follicular lymphoma identifies a risk locus at 6p21.32. Nat. Genet. 2010, 42, 661-664. [CrossRef]

51. Qiao, Y.; Zhou, Y.; Wu, C.; Zhai, K.; Han, X.; Chen, J.; Tian, X.; Chang, J.; Lu, Z.; Zhang, B.; et al. Risk of genome-wide association study-identified genetic variants for non-Hodgkin lymphoma in a Chinese population. Carcinogenesis 2013, 34, 1516-1519. [CrossRef]

52. Cerhan, J.R.; Fredericksen, Z.S.; Novak, A.J.; Ansell, S.M.; Kay, N.E.; Liebow, M.; Doğan, A.; Cunningham, J.M.; Wang, A.H.; Witzig, T.E.; et al. A Two-Stage Evaluation of Genetic Variation in Immune and Inflammation Genes with Risk of Non-Hodgkin Lymphoma Identifies New Susceptibility Locus in 6p21.3 Region. Cancer Epidemiol. Biomark. Prev. 2012, 21, 1799-1806. [CrossRef] [PubMed]

53. Skibola, C.F.; Conde, L.; Foo, J.N.; Riby, J.; Humphreys, K.; Sillé, F.C.M.; Darabi, H.; Sanchez, S.; Hjalgrim, H.; Liu, J.; et al. A meta-analysis of genome-wide association studies of follicular lymphoma. BMC Genom. 2012, 13, 516. [CrossRef] [PubMed]

54. Skibola, C.F.; Berndt, S.I.; Vijai, J.; Conde, L.; Wang, Z.; Yeager, M.; De Bakker, P.I.; Birmann, B.M.; Vajdic, C.M.; Foo, J.N.; et al. Genome-wide Association Study Identifies Five Susceptibility Loci for Follicular Lymphoma outside the HLA Region. Am. J. Hum. Genet. 2014, 95, 462-471. [CrossRef]

55. Skibola, C.F.; Bracci, P.M.; Halperin, E.; Conde, L.; Craig, D.W.; Agana, L.; Iyadurai, K.; Becker, N.; Brookes-Wilson, A.; Curry, J.D.; et al. Genetic variants at 6p21.33 are associated with susceptibility to follicular lymphoma. Nat. Genet. 2009, 41, 873-875. [CrossRef]

56. Di Bernardo, M.C.; Crowther-Swanepoel, D.; Broderick, P.; Webb, E.; Sellick, G.; Wild, R.; Sullivan, K.; Vijayakrishnan, J.; Wang, Y.; Pittman, A.M.; et al. A genome-wide association study identifies six susceptibility loci for chronic lymphocytic leukemia. Nat. Genet. 2008, 40, 1204-1210. [CrossRef]

57. Crowther-Swanepoel, D.; Broderick, P.; Di Bernardo, M.C.; Dobbins, S.E.; Torres, M.P.; Mansouri, M.; Ruiz-Ponte, C.; Enjuanes, A.; Rosenquist, R.; Carracedo, A.; et al. Common variants at 2q37.3, 8q24.21, 15q21.3 and 16q24.1 influence chronic lymphocytic leukemia risk. Nat. Genet. 2010, 42, 132-136. [CrossRef] 
58. Slager, S.L.; Benavente, Y.; Blair, A.; Vermeulen, R.; Cerhan, J.R.; Costantini, A.S.; Monnereau, A.; Nieters, A.; Clavel, J.; Call, T.G.; et al. Medical History, Lifestyle, Family History, and Occupational Risk Factors for Chronic Lymphocytic Leukemia/Small Lymphocytic Lymphoma: The InterLymph Non-Hodgkin Lymphoma Subtypes Project. J. Nat. Cancer Inst. Monogr. 2014, 2014, 41-51. [CrossRef]

59. Sampson, J.N.; Wheeler, W.A.; Yeager, M.; Panagiotou, O.A.; Wang, Z.; Berndt, S.I.; Lan, Q.; Abnet, C.C.; Amundadottir, L.T.; Figueroa, J.D.; et al. Analysis of Heritability and Shared Heritability Based on Genome-Wide Association Studies for Thirteen Cancer Types. J. Nat. Cancer Inst. 2015, 107, djv279. [CrossRef]

60. Gragert, L.; Fingerson, S.; Albrecht, M.; Maiers, M.; Kalaycio, M.; Hill, B.T. Fine-mapping of HLA associations with chronic lymphocytic leukemia in US populations. Blood 2014, 124, 2657-2665. [CrossRef]

61. Machulla, H.; Schaaf, A.; Kujat, G.; Langner, J. Association of chronic lymphocytic leukemia with specific alleles of the HLADR4:DR53:DQ8 haplotype in German patients. Int. J. Cancer 2001, 92, 203-207. [CrossRef]

62. Slager, S.L.; Rabe, K.G.; Achenbach, S.J.; Vachon, C.M.; Goldin, L.R.; Strom, S.S.; Lanasa, M.C.; Spector, L.G.; Rassenti, L.Z.; Leis, J.F.; et al. Genome-wide association study identifies a novel susceptibility locus at 6p21.3 among familial CLL. Blood 2011, 117, 1911-1916. [CrossRef] [PubMed]

63. Mueller, L.P.; Machulla, H.K.G. Increased frequency of homozygosity for HLA class II loci in female patients with chronic lymphocytic leukemia. Leuk. Lymphoma 2002, 43, 1013-1019. [CrossRef] [PubMed]

64. Slager, S.L.; Skibola, C.F.; Di Bernardo, M.C.; Conde, L.; Broderick, P.; McDonnell, S.K.; Goldin, L.R.; Croft, N.; Holroyd, A.; Harris, S.; et al. Common variation at 6p21.31 (BAK1) influences the risk of chronic lymphocytic leukemia. Blood 2012, 120, 843-846. [CrossRef]

65. Berndt, S.I.; Skibola, C.F.; Joseph, V.; Camp, N.J.; Nieters, A.; Wang, Z.; Cozen, W.; Monnereau, A.; Wang, S.S.; Kelly, R.S.; et al. Genome-wide association study identifies multiple risk loci for chronic lymphocytic leukemia. Nat. Genet. 2013, 45, 868-876. [CrossRef] [PubMed]

66. Speedy, H.E.; Di Bernardo, M.C.; Sava, G.P.; Dyer, M.J.S.; Holroyd, A.; Wang, Y.; Sunter, N.J.; Mansouri, L.; Juliusson, G.; Smedby, K.E.; et al. A genome-wide association study identifies multiple susceptibility loci for chronic lymphocytic leukemia. Nat. Genet. 2014, 46, 56-60. [CrossRef]

67. Berndt, S.I.; Camp, N.J.; Skibola, C.F.; Vijai, J.; Wang, Z.; Gu, J.; Nieters, A.; Kelly, R.S.; Smedby, K.E.; Monnereau, A.; et al. Meta-analysis of genome-wide association studies discovers multiple loci for chronic lymphocytic leukemia. Nat. Commun. 2016, 7, 10933. [CrossRef]

68. Law, P.J.; Berndt, S.I.; Speedy, H.E.; Camp, N.J.; Sava, G.P.; Skibola, C.F.; Holroyd, A.; Vijai, J.; Sunter, N.J.; Nieters, A.; et al. Genome-wide association analysis implicates dysregulation of immunity genes in chronic lymphocytic leukaemia. Nat. Commun. 2017, 8, 14175. [CrossRef]

69. Law, P.J.; Sud, A.; Mitchell, J.S.; Henrion, M.; Orlando, G.; Lenive, O.; Broderick, P.; Speedy, H.E.; Johnson, D.C.; Kaiser, M.; et al. Genome-wide association analysis of chronic lymphocytic leukaemia, Hodgkin lymphoma and multiple myeloma identifies pleiotropic risk loci. Sci. Rep. 2017, 7, 41071. [CrossRef]

70. Crowther-Swanepoel, D.; Mansouri, M.; Enjuanes, A.; Vega, A.; Smedby, K.E.; Ruiz-Ponte, C.; Jurlander, J.; Juliusson, G.; Montserrat, E.; Catovsky, D.; et al. Verification that common variation at 2q37.1, 6p25.3, 11q24.1, 15q23, and 19q13.32 influences chronic lymphocytic leukaemia risk. Br. J. Haematol. 2010, 150, 473-479. [CrossRef]

71. Sava, G.P.E.; Speedy, H.; Di Bernardo, M.C.; Dyer, M.J.S.; Holroyd, A.; Sunter, N.J.; Marr, H.; Mansouri, L.; Deaglio, S.; Karabon, L.; et al. Common variation at 12q24.13 (OAS3) influences chronic lymphocytic leukemia risk. Leukemia 2014, 29, 748-751. [CrossRef]

72. Vijai, J.; Wang, Z.; Berndt, S.I.; Skibola, C.F.; Slager, S.L.; De Sanjose, S.; Melbye, M.; Glimelius, B.; Bracci, P.M.; Conde, L.; et al. A genome-wide association study of marginal zone lymphoma shows association to the HLA region. Nat. Commun. $2015,6,5751$. [CrossRef] [PubMed]

73. Vijai, J.; Wang, Z.; Berndt, S.I.; Slager, S.L.; Cerhan, J.R.; Skibola, C.; Wang, S.S.; Brooks-Wilson, A.R.; De Sanjosé, S.; Bracci, P.M.; et al. Abstract 5071: A genome-wide association study suggests evidence of variants at 6p21.32 associated with marginal zone lymphoma. In Proceedings of the Epidemiology, American Association for Cancer Research (AACR), New Orleans, LA, USA, 20-23 September 2014; Volume 74, p. 5071.

74. Bracci, P.M.; Benavente, Y.; Turner, J.J.; Paltiel, O.; Slager, S.L.; Vajdic, C.M.; Norman, A.D.; Cerhan, J.R.; Chiu, B.C.H.; Becker, N.; et al. Medical History, Lifestyle, Family History, and Occupational Risk Factors for Marginal Zone Lymphoma: The InterLymph Non-Hodgkin Lymphoma Subtypes Project. J. Nat. Cancer Inst. Monogr. 2014, 2014, 52-65. [CrossRef] [PubMed]

75. LaBreche, K.; Daniau, M.; Sud, A.; Law, P.J.; Royer-Perron, L.; Holroyd, A.; Broderick, P.; Went, M.; Benazra, M.; Ahle, G.; et al. A genome-wide association study identifies susceptibility loci for primary central nervous system lymphoma at 6p25.3 and 3p22.1: A LOC Network study. Neuro-Oncol. 2019, 21, 1039-1048. [CrossRef] [PubMed]

76. Garcilazo-Reyes, Y.; Ibáñez-Juliá, M.-J.; Hernández-Verdin, I.; Nguyen-Them, L.; Younan, N.; Houillier, C.; Hoang-Xuan, K.; Alentorn, A. Treating central nervous system lymphoma in the era of precision medicine. Expert Rev. Precis. Med. Drug Dev. 2020, 5, 275-281. [CrossRef]

77. Yu, C.C.; Chen, L.C.; Huang, C.Y.; Lin, V.C.; Lu, T.L.; Lee, C.H.; Huang, S.; Bao, B.Y. Genetic association analysis identifies a role for ANO5 in prostate cancer progression. Cancer Med. 2020, 9, 2372-2378. [CrossRef] 
78. Tan, D.E.K.; Foo, J.N.; Bei, J.X.; Chang, J.; Peng, R.; Zheng, X.; Wei, L.; Huang, Y.; Lim, W.Y.; Li, J.; et al. Genome-wide association study of B cell non-Hodgkin lymphoma identifies 3q27 as a susceptibility locus in the Chinese population. Nat. Genet. 2013, 45, 804-807. [CrossRef]

79. Slager, S.L.; Camp, N.J.; Conde, L.; Shanafelt, T.D.; Achenbach, S.J.; Rabe, K.G.; Kay, N.E.; Novak, A.J.; Call, T.G.; Bracci, P.M.; et al. Common variants within 6p21.31 locus are associated with chronic lymphocytic leukaemia and, potentially, other non-Hodgkin lymphoma subtypes. Br. J. Haematol. 2012, 159, 572-576. [CrossRef]

80. Casellas, R.; Basu, U.; Yewdell, W.T.; Chaudhuri, W.T.Y.J.; Robbiani, D.F.; Di Noia, J.M. Mutations, kataegis and translocations in B cells: Understanding AID promiscuous activity. Nat. Rev. Immunol. 2016, 16, 164-176. [CrossRef]

81. Kohli, R.M.; Maul, R.W.; Guminski, A.F.; McClure, R.L.; Gajula, K.S.; Saribasak, H.; McMahon, M.A.; Siliciano, R.F.; Gearhart, P.J.; Stivers, J.T. Local Sequence Targeting in the AID/APOBEC Family Differentially Impacts Retroviral Restriction and Antibody Diversification. J. Biol. Chem. 2010, 285, 40956-40964. [CrossRef]

82. Kasar, S.; Kim, J.; Improgo, R.; Tiao, G.; Polak, P.; Haradhvala, N.; Lawrence, M.S.; Kiezun, A.; Fernandes, S.M.; Bahl, S.; et al. Whole-genome sequencing reveals activation-induced cytidine deaminase signatures during indolent chronic lymphocytic leukaemia evolution. Nat. Commun. 2015, 6, 8866. [CrossRef]

83. Holmes, A.B.; Corinaldesi, C.; Shen, Q.; Kumar, R.; Compagno, N.; Wang, Z.; Nitzan, M.; Grunstein, E.; Pasqualucci, L.; DallaFavera, R.; et al. Single-cell analysis of germinal-center B cells informs on lymphoma cell of origin and outcome. J. Exp. Med. 2020, 217, e20200483. [CrossRef] [PubMed] 